\title{
A Numerical Test of Padé Approximation for Some Functions with Singularity
}

\author{
Hiroaki S. Yamada ${ }^{1}$ and Kensuke S. Ikeda ${ }^{2}$ \\ ${ }^{1}$ Yamada Physics Research Laboratory, Aoyama 5-7-14-205, Niigata 950-2002, Japan \\ ${ }^{2}$ Department of Physics, Ritsumeikan University, Noji-higashi 1-1-1, Kusatsu 525, Japan
}

Correspondence should be addressed to Hiroaki S. Yamada; hyamada@uranus.dti.ne.jp

Received 17 July 2014; Accepted 14 October 2014; Published 20 November 2014

Academic Editor: Don Hong

Copyright (C) 2014 H. S. Yamada and K. S. Ikeda. This is an open access article distributed under the Creative Commons Attribution License, which permits unrestricted use, distribution, and reproduction in any medium, provided the original work is properly cited.

\begin{abstract}
The aim of this study is to examine some numerical tests of Pade approximation for some typical functions with singularities such as simple pole, essential singularity, brunch cut, and natural boundary. As pointed out by Baker, it was shown that the simple pole and the essential singularity can be characterized by the poles of the Padé approximation. However, it was not fully clear how the Padé approximation works for the functions with the branch cut or the natural boundary. In the present paper, it is shown that the poles and zeros of the Padé approximated functions are alternately lined along the branch cut if the test function has branch cut, and poles are also distributed around the natural boundary for some lacunary power series and random power series which rigorously have a natural boundary on the unit circle. On the other hand, Froissart doublets due to numerical errors and/or external noise also appear around the unit circle in the Padé approximation. It is also shown that the residue calculus for the Padé approximated functions can be used to confirm the numerical accuracy of the Padé approximation and quasianalyticity of the random power series.
\end{abstract}

\section{Introduction}

Padé approximation was introduced in mathematics by Hermite and Padé and it has been used in physics for more than 40 years ago [1-3]. In particular, there have been several important examples in physics, to which the Padé approximation was applied, such as summation of the divergent Rayleigh-Schrodinger perturbation series in scattering theory [4], critical phenomena in statistical physics [5-9], denoising from noisy data of time-series [10-14], and detection of singularity of phase space trajectories of Hamiltonian dynamical systems [15-19].

Mathematically, the Padé approximation can be used to estimate analyticity of functions. Indeed, the Padé approximation is usually superior to the truncated Taylor expansions when the original function contains any singularity. Let us consider a simple example. The function $f(z)=$ $\sqrt{(1+2 z) /(1+z)}$ has brunch points at $z=-1$ and $z=-1 / 2$. The domain of convergence is $|z|<1 / 2$. Nevertheless, we can obtain an exact solution $\sqrt{ } 2=1.4142$ for $z \rightarrow \infty$ when we apply [3 | 3] diagonal Padé approximation to the function
(See Section 3.3 for more details on this example) Although the mathematical validity of the Padé approximation has not been exactly proved yet, the Padé approximation is practically very useful to continue a singular function beyond the domain of convergence.

Let us consider a critical phenomenon for Ising model as a simple example in the statistical physics $[5,7,8]$. We assume that at the critical point $u=u_{c}$ the exact magnetic susceptibility $\chi$ has a singularity as

$$
\chi \sim\left(u-u_{c}\right)^{-\gamma}
$$

where $u$ is a function of temperature and interactions and so on. In this case we sometimes use the logarithmic derivative of $\chi$ when we estimate the critical point $u=u_{c}$ and the critical exponent $\gamma$ as a pole-type singularity as

$$
\frac{d \log \chi}{d u}=\frac{\chi^{\prime}}{\chi}=\frac{\gamma}{\left(u-u_{c}\right)}
$$


where $\chi^{\prime}$ denotes the derivative with respect to the variable $u$. In the low temperature expansion for the magnetic susceptibility $\chi$ with some coefficients $a_{n}$, we assume that the approximated susceptibility $\chi_{N}$ and the logarithmic derivative can be obtained as follows:

$$
\chi \sim \chi_{N}=\sum_{n=0}^{N} a_{n} u^{n}, \quad \frac{\chi^{\prime}}{\chi} \sim \sum_{n=0}^{N} b_{n} u^{n} .
$$

Then we can estimate the critical point $u=u_{c}$ and the critical exponent $\gamma$ by the Padé approximation to the truncated expansion $\chi_{N}$. Note that the singularity on $|u|=u_{c}$ will be infinitely differentiable if the coefficients $a_{n}$ fall off sufficiently rapidly.

In general, analytic continuation over the singular point is possible along any other path in complex plane even if the function diverges at the singular point determining the radius of convergence, as seen in the above singularity of the Ising model. Therefore, the Padé approximation is useful to improve the convergence of the power series and approximate the exact solution.

Furthermore, the Padé approximation has been used to investigate convergence of Fourier series $[2,3]$ and the breakdown of KAM curves in complex plane for Hamiltonian map systems, which is described as the analytic domains of Lindstedt series for standard map [15-19].

In addition, we can see an interesting example concerning the Padé approximation in noisy data analysis [10-14]. The power series with finite random coefficient "almost always" has a natural boundary on the unit circle in the complex plane [20-24]. In a finite time-series, Froissart has shown that a natural boundary generated by the random time-series is approximated by doublets of poles and zeros (Froissart doublets) of the Padé approximated function, which are surrounding the vicinity of the unit circle. Taking advantage of the characteristic, the Padé approximation has been used in order to remove the noise and extract the true poles associated with damping modes from the observed noisy timeseries.

The main purpose of the present paper is to investigate whether the Padé approximation is numerically useful for detecting the singularity of some test functions. In particular, we examine the usefulness for the functions with a natural boundary such as a lacunary power series and a random power series $[2,13,18]$.

The organization of the paper is as follows. In Section 2, we give a brief explanation of the Padé approximation and some important reminders in the numerical calculation. In Section 3, we present some numerical results of the Padé approximation for test functions with branch cut, essential singularity. We also try to apply the Padé approximation to an entire function. In Section 4, application of the Padé approximation to some lacunary series which is known to have a natural boundary is given. In Section 5, numerical results of the application to random power series with a natural boundary and some test functions with random noise are also shown. In Section 6, we discuss the residue calculus for the Padé approximated functions to confirm the numerical errors and quasianalyticity of the random power series. In the last section, we give summary and discussion.

In Appendix A, the general result for Fibonacci generator used in Section 3.2 is given. In Appendix B, some theorems concerning zeros of polynomials are given. In Appendix C, some mathematical theorems for lacunary series, which are useful in reading the main text, are summarized. In Appendix D, exact Padé approximated functions to some lacunary power series with a natural boundary are given. Residue analysis for quasianalytic functions of Carleman class is given in Appendix E. Furthermore, some theorems concerning the random power series are given in Appendix F.

\section{Padé Approximation}

In this section, we give preliminary instructions to the Padé approximation and some important reminders on the numerical calculation.

For a given function $f(z)$, a truncated Taylor expansion $f^{[N]}(z)$ of order $N$ about zero is given as

$$
f(z) \sim f^{[N]}(z)=\sum_{n=0}^{N} c_{n} z^{n}
$$

where $c_{n}$ denotes the coefficients of the Taylor expansion. Padé approximation is more accurate approximation for $f(z)$ up to order $O\left(z^{N}\right)$ than the Taylor expansion. The Padé approximation is a rational function, namely, a ratio of two polynomials, which agrees with the highest possible order $O\left(z^{N}\right)$ with a truncated polynomial $f^{[N]}(z)$ as follows:

$$
\begin{aligned}
f^{[N]}(z) & =\frac{a_{0}+a_{1} z+a_{2} z^{2}+\cdots a_{L} z^{L}}{1+b_{1} z+b_{2} z^{2}+\cdots b_{M} z^{M}} \\
& \equiv \frac{P_{L}(z)}{Q_{M}(z)} \equiv f^{[L \mid M]}(z),
\end{aligned}
$$

where $P_{L}(z)$ is a polynomial of degree less than or equal to $L$ and $Q_{M}(z)$ is a polynomial of degree less than or equal to $M$. Note that $b_{0}=1$ (normalized) here. A unique approximation can be specified for all choices of $M$ and $L$ such that $N=$ $L+M$ when it exists. The coefficients $\left\{a_{n}\right\},\left\{b_{n}\right\}$ can be obtained from the condition that the first $(L+M+1)$ terms vanish in the Taylor series in (4). The difference between the Padé approximation and the original function satisfies the following equation:

$$
f(z)-f^{[L \mid M]}(z)=O\left(z^{L+M+1}\right) .
$$

In this paper, we sometimes use " $\left[\begin{array}{lll}L & M\end{array}\right]$ Padé approximation" or " $[L \mid M]$ Padé approximated function" for $f^{[L \mid M]}(z)$. Solving the problem in (6) is called a linear Teoplitz problem which is generally ill-conditioned. We used full LU decomposition for the Teoplitz matrix in the problem as well as iterative improvement in order to eliminate the ill-posed problem $[25,26]$. In addition, hereafter, we use the diagonal Padé approximation, that is, $L=M$, of order $M \leq 65$ 
to estimate the singularity of the test functions because of the convergence and limitation due to the round-off errors and other sources of numerical errors. Here, the singularity of the function $f(z)$ is approximated by configuration of the poles and zeros of the $[M \mid M]$ order diagonal Padé approximated function. As mentioned in Introduction, in general, the Padé approximations are useful for representing unknown functions with possible poles. The application of the diagonal Padé approximation is insured for the functions with isolated singular points and rational-type functions. However, it is not fully clarified how the poles and zeros of the Padé approximated function describe brunch cut and natural boundary [27-29].

Generally, the magnitude of the residues associated with the spurious poles is much smaller than that with the true poles, and they are close to machine precision. Very recently, Gonnet et al. suggested an efficient algorithm for the Padé approximations [30,31]. The algorithm detects and eliminates the spurious pole-zero pairs caused by the rounding errors by means of singular value decomposition for the Teoplitz matrix.

Before closing this section, we list up some important reminders when we numerically apply the Padé approximation to unknown functions as follows.

(1) More accurate calculation becomes possible by scaling the expansion variable $z$ if there is a simple pole with large magnitude $\rho(\gg 1)$. That is, we should change the order of radius of convergence into $O(1)$ by the scaling the expansion variable as $z \rightarrow z / \rho$ in order to keep the numerical accuracy. This procedure is effective when we apply the Padé approximation to exponentially decaying coefficients $\left\{a_{n}\right\}$ with fluctuation.

(2) Poles (i.e., roots of $P_{M}(z)=0$ ) and zeros (i.e., roots of $Q_{M}(z)=0$ ) are sometimes cancelled (zero-pole ghost pairs). We can remove the effects of the ghost pairs and confirm the singularity of the functions by using the residue analysis of the Padé approximated function.

(3) Poles and zeros of the Padé approximation to the truncated random power series accumulate around the unit circle as Froissart doublets. It is difficult to distinguish whether the poles of the Padé approximation originated from a natural boundary of the original function or the natural boundary generated by the numerical error and/or noise. Therefore, the numerical accuracy will be important to determine the coefficients of the Padé approximation.

(4) In general, the denominators of the diagonal Padé approximated functions to the lacunary power series and the random power series become lacunary and random polynomials, respectively. Accordingly, the distribution of the poles and zeros of the approximated functions are similar to the distribution of the zeros corresponding to the original lacunary power polynomial and random power polynomial. In particular, it is well known that the zeros of the random polynomials uniformly distribute about the unit circle (see Appendix B).

\section{Examples of Padé Approximation for Some Functions}

In this section we investigate the configuration of the poles and zeros of the Padé approximation to some test functions with singularity.

3.1. Comparison of Padé Approximation with Taylor Expansion. First, we try the Padé approximation to the following test function $f_{1}(z)$ with a brunch point at $z=-1$ :

$$
f_{1}(z)=\frac{\log (1+z)}{z}
$$

The truncated Taylor expansion of the order $N=4$ around $z=0$ is

$$
f_{1}^{[4]}(z)=1-\frac{1}{2} z+\frac{1}{3} z^{2}-\frac{1}{4} z^{3}+\frac{1}{5} z^{4} .
$$

The [2|2] Padé approximation is given as

$$
f_{1}^{[2 \mid 2]}(z)=\frac{1+(7 / 10) z+(1 / 30) z^{2}}{1+(6 / 5) z+(3 / 10) z^{2}} .
$$

Figure 1 shows the approximated functions, the truncated Taylor series $f_{1}^{[4]}(z)$, and the original test function $f_{1}(z)$. The approximated function $f_{1}^{[4]}(z)$ by the truncated Taylor expansion converges only within $|z|<1$ and deviates from the exact function $f_{1}(z)$ for $|z|>1$. On the other hand, it follows that the Padé approximated function $f_{1}^{[2 \mid 2]}(z)$ well approximates the original function $f_{1}(z)$ with very high precession even beyond the radius of convergence up to $\operatorname{Re} z=$ $x \sim 10$. Therefore, it is found that the divergent power series expansion (Taylor expansion) does still contain information about the original function outside the convergence radius, and rearranging the coefficients of the expansion into the Padé approximation recovers the information. As a result, the conversion from the Taylor form to the Padé form usually accelerates the convergence and often allows good accuracy even outside the radius of convergence of the power series.

3.2. Padé Approximation for Fibonacci Generating Function. Let us consider the Fibonacci generating function $f_{F}(z)$, where Fibonacci sequence $\left\{F_{n}\right\}$ is encoded in the power series as the coefficients. The Fibonacci sequence $\left\{F_{n}\right\}$ is given by the following recursion relation:

$$
F_{n}=F_{n-1}+F_{n-2} \quad(n \geq 2),
$$

where $F_{0}=0$ and $F_{1}=1$. Then the Fibonacci generating function $f_{F}(z)$ becomes 


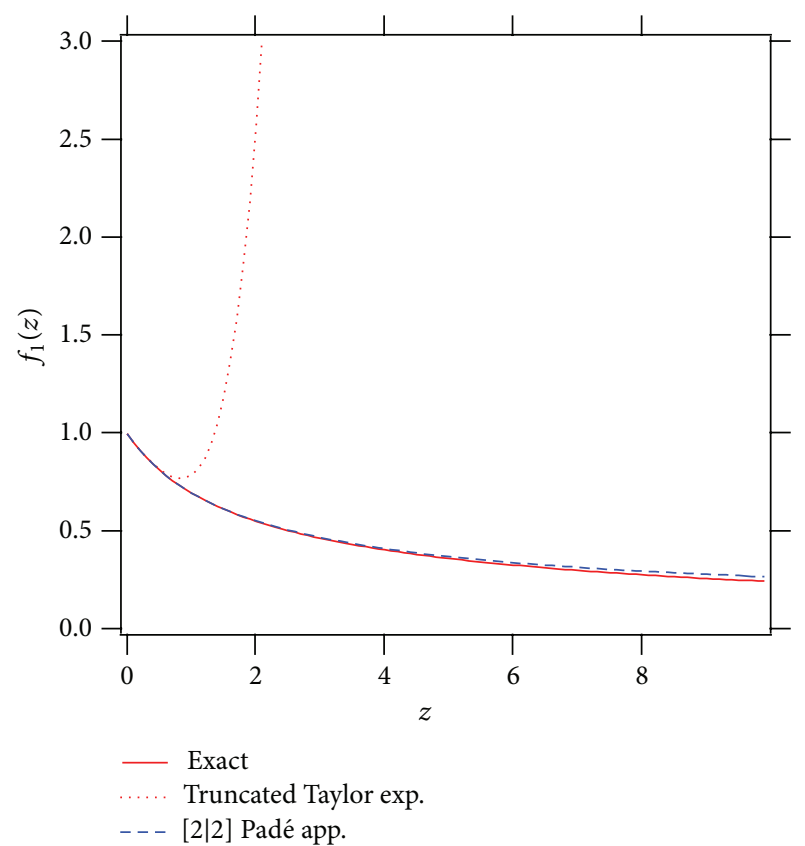

FIgUre 1: The Padé approximated function $f_{1}^{[2 \mid 2]}(z)$, the truncated Taylor series $f_{1}^{[4]}(z)$, and the original test function $f_{1}(z)$.

$$
\begin{aligned}
f_{F}(z) & =\sum_{n=0}^{\infty} F_{n} z^{n} \\
& =\frac{z}{1-z-z^{2}} \\
& =\frac{1}{\sqrt{5}} \frac{1}{1-\phi^{+} z}-\frac{1}{\sqrt{5}} \frac{1}{1-\phi^{-} z}
\end{aligned}
$$

where $\phi^{+} \equiv((1+\sqrt{5}) / 2)(=1.61803 \ldots)$ and $\phi^{-} \equiv((1-$ $\sqrt{5}) / 2)(=-0.61803 \ldots)$. The generating function has poles at $z=\phi^{+}$and $z=\phi^{-}$. Generating functions by more general recursion relation is given in Appendix A.

It should be noted that the diagonal Padé approximation $f_{F}^{[N / 2 \mid N / 2]}(z)$ for the truncated Fibonacci generation function $f_{F}^{[N]}(z)=\sum_{n=0}^{N} F_{n} z^{n}$ has the following form:

$$
f_{F}^{[N / 2 \mid N / 2]}(z)=\frac{z}{1-z-z^{2}}+O\left(z^{N+1}\right),
$$

for even number $N \geq 2$. This means that the diagonal Padé approximation can detect the exact poles of the generating function irrespective of the order.

3.3. Examples of Some Test Functions with Pole, Brunch Cut, and Essential Singularity. In this subsection, we use some test functions in applying of the Padé approximation:

$$
\begin{gathered}
f_{2}(z)=e^{-z} \\
f_{3}(z)=\sqrt{\frac{1+2 z}{1+z}} \\
f_{4}(z)=e^{-z /(1+z)}, \\
f_{5}(z)=\tan z^{4} .
\end{gathered}
$$

Here, $f_{2}(z)$ has no singularity for $|z|<\infty, f_{3}(z)$ has a brunch cut along a line on $[-1,-1 / 2]$, and $f_{4}(z)$ has an essential singularity at $z=-1 . f_{5}(z)$ has eight poles at points on the unit circle $z=\exp \{i \pi(m / 4)\}(m=0,1,2, \ldots, 7)$.

First, let us apply Padé approximation to $f_{2}(z)$. In this case the explicit form of the Padé approximated function can be obtained in the following form [33]:

$$
\begin{gathered}
P_{M}(z)=\sum_{k=0}^{M} \frac{(2 M-k) ! M !}{(2 M) ! k !(M-k) !}(-z)^{k}, \\
Q_{M}(z)=\sum_{k=0}^{M} \frac{(2 M-k) ! M !}{(2 M) ! k !(M-k) !} z^{k} .
\end{gathered}
$$

Note that the coefficients of the numerator $P_{M}(z)$ have always alternative sign, and the zeros and poles of the Padé approximated function are symmetrical to the imaginary axis with each other because $P_{M}(z)=Q_{M}(-z)$. Figure 2(a) shows the numerical results in the complex $z$-plane. All poles are on the left half-plane $\operatorname{Re} z>0$, and all zeros are on the right half-plane $\operatorname{Re} z<0$. The poles and zeros of the Padé approximated functions for the regular function $f_{2}(z)$ go infinite and disappear as $M \rightarrow \infty$ because the function $f_{2}(z)$ is an entire function.

In Figure 2(b), distribution of the zeros and poles of the Padé approximated function to $f_{3}(z)$ is shown. The poles and zeros make a line alternately between the two branch points of the $f_{3}(z) ; z=-1$ and $z=-1 / 2$.

Figure 2(c) shows the distribution of the zeros and poles of the Padé approximation to $f_{4}(z)$. The Padé approximation clusters the poles and zeros at the singular point for $f_{4}(z)$. As $M \rightarrow \infty$ the poles and zeros approach the singular point $z=-1$ that reflects the essential singularity of the original function $f_{4}(z)$.

Figure 3(a) shows the poles and zeros of the Padé approximated function to the test function $f_{5}(z)$. The poles and zeros are alternatively distributed in eight directions from the origin. It seems that the distance between the poles and/or zeros on the same line becomes small as they approach the location of the true poles. Some "spurious poles" appear around the unit circle with the increase of the order of the Padé approximation, as seen in Figure 3(b), which are irrelevant poles due to insufficient numerical accuracy. It is found that the numerical accuracy of the Padé approximation fails for the higher order of the Padé approximation. We discuss the spurious poles in Sections 4 and 5 again.

\section{Natural Boundary of Lacunary Power Series}

In this section, we examine the applicability of the Padé approximation to investigate the analyticity of some well-known test functions with a natural boundary on the unit circle $|z|=1$. This will provide a preliminary information about what occurs in the Padé approximated functions for some 


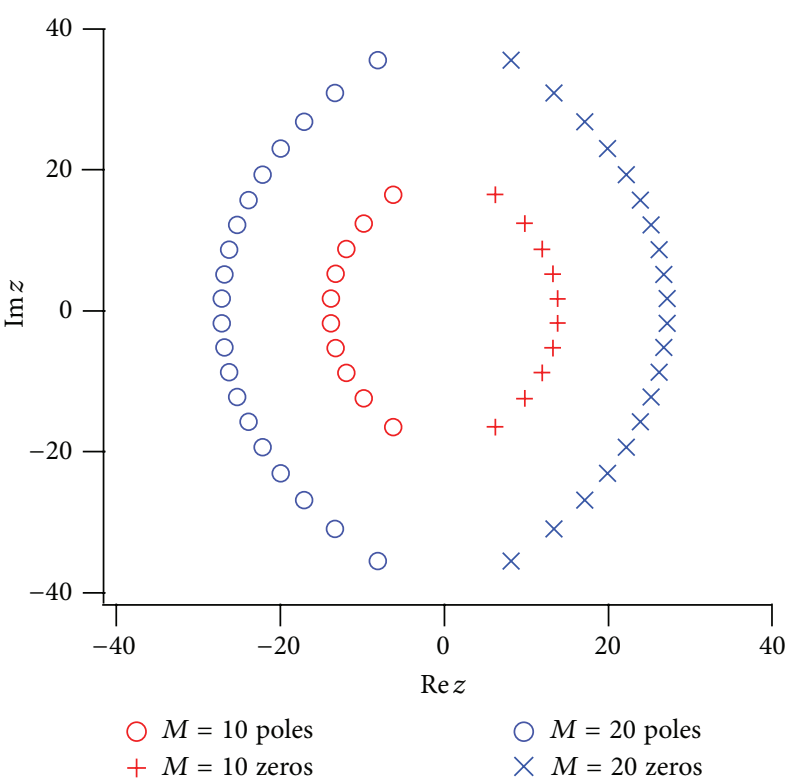

(a)

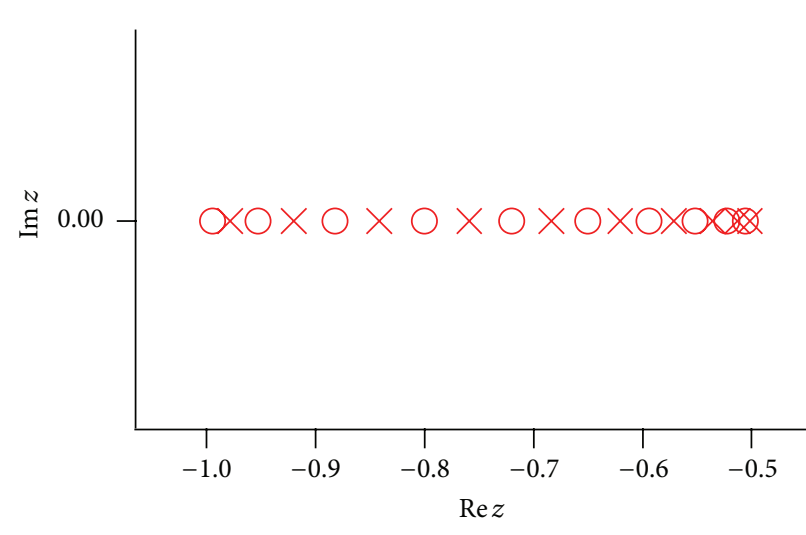

$\bigcirc$ Poles

X Zeros

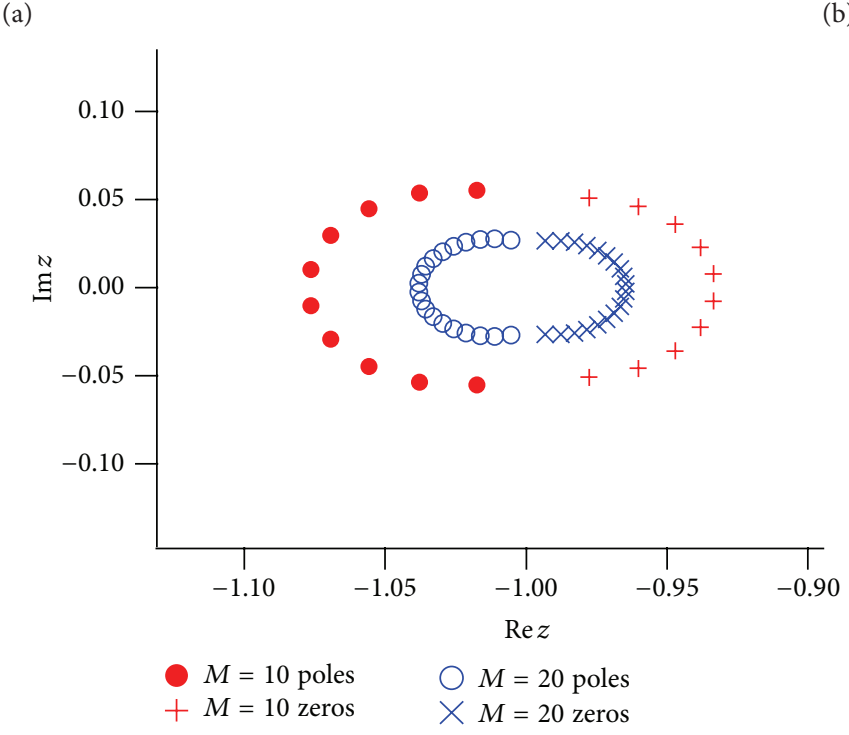

(b)

(c)

Figure 2: Distributions of poles $(\bullet, \bigcirc)$ and zeros $(+, \times)$ of the $[M \mid M]$ diagonal Padé approximated functions for some test functions: (a) $f_{2}(z),\left(\right.$ b) $f_{3}(z)$, and (c) $f_{4}(z)$.

test functions with a natural boundary. Indeed, we do know only a very few numerical examples which have a natural boundary and allow an exact diagonal Padé approximation.

The following are famous lacunary power series with a natural boundary on the unit circle $|z|=1, f_{\mathrm{Jac}}(z)=\sum_{n=0}^{\infty} z^{2^{n}}$, $f_{\mathrm{Wie}}(z)=\sum_{n=0}^{\infty} z^{n !}$, and $f_{\mathrm{Kro}}(z)=\sum_{n=0}^{\infty} z^{n^{2}}$, where the $f_{\mathrm{Jac}}(z)$, $f_{\text {Wie }}(z)$, and $f_{\text {Kro }}(z)$ are called after Jacobi, Weierstrass, and Kronecker. Some theorems for the lacunary series with a natural boundary are given in Appendix C [20-22].

Here, we use polar-form $f_{r}(\theta)$ for the function $f(z)$ by changing the variable, that is, $z=r e^{i \theta}$, in order to simply display the functions as

$$
f_{r}(\theta)=f\left(z=r e^{i \theta}\right)=\sum_{n=0}^{\infty} c_{n}\left(r e^{i \theta}\right)^{n} .
$$

Then, note that the modulus $r$ works as a convergence factor of the series because it well converges for $r<1$. Typically we take $r=1$ on the unit circle or $r=0.98$ inside the circle in the following numerical calculations.

4.1. Example 1: Jacobi Lacunary Series. We try to apply Padé approximation to the function $f_{\mathrm{Jac}}(z)$ with a natural 


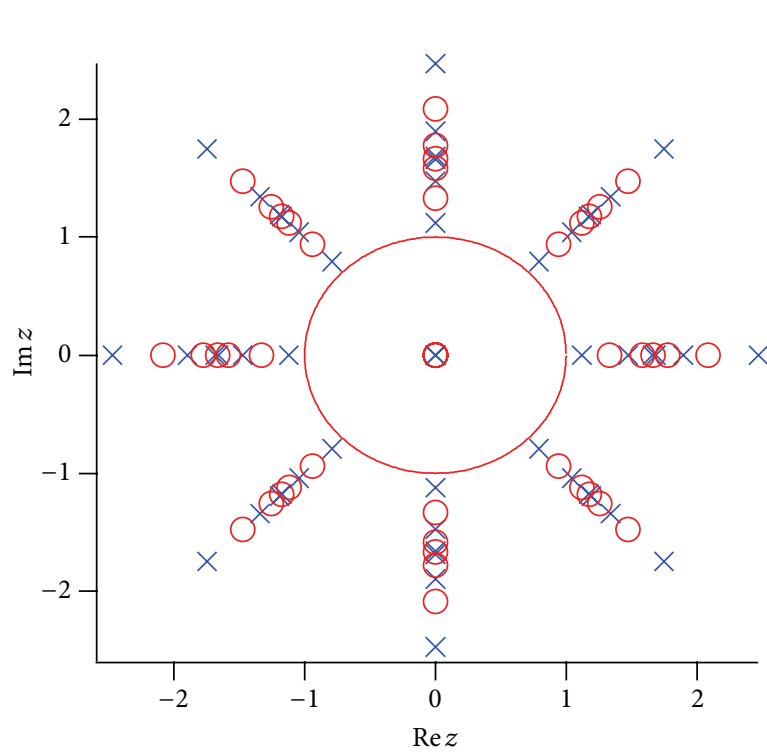

(a)

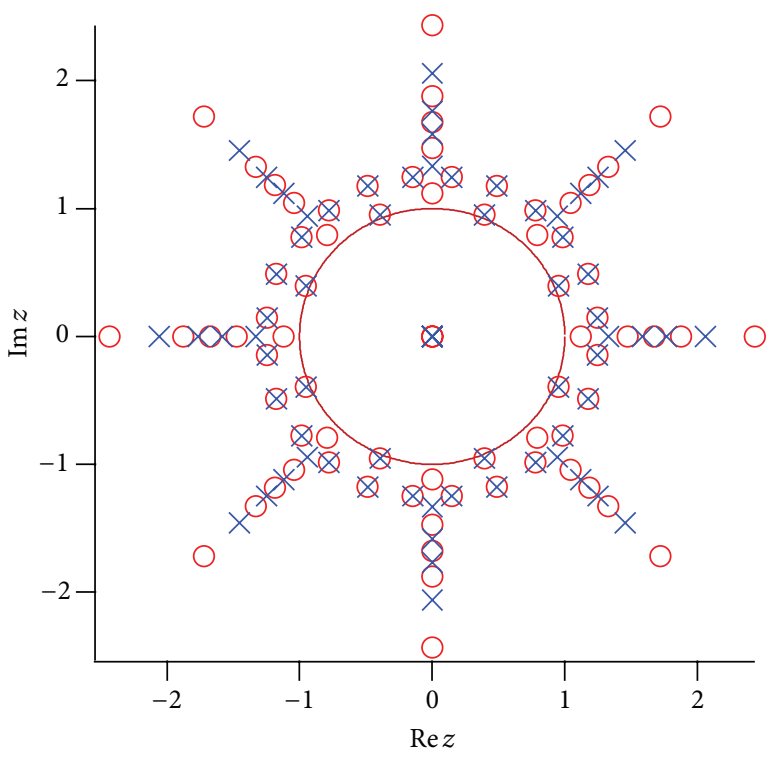

(b)

Figure 3: Distributions of poles $(\bigcirc)$ and zeros $(\times)$ of the $[M \mid M]$ diagonal Padé approximated functions for the test function $f_{5}(z)$. (a) The [50 | 50] Padé approximation. (b) The [75| 75] Padé approximation. The unit circle is drawn to guide the eye.

boundary on the unit circle $|z|=1$. The Padé approximated function exactly has the following form:

$$
\begin{aligned}
f_{\mathrm{Jac}}^{\left[2^{N}\right]}(z) & \sim f_{\mathrm{Jac}}^{\left[2^{N-1} \mid 2^{N-1}\right]}(z) \\
& =\frac{A_{\mathrm{Jac}}^{N}(z)}{1+\sum_{k=0}^{N-2} z^{2^{k}}-z^{2^{N-1}}},
\end{aligned}
$$

where the explicit form of the numerator $A_{\mathrm{Jac}}^{N}(z)$ is given in Appendix D. Accordingly, the poles of the $\left[2^{N-1} \mid 2^{N-1}\right]$ Padé approximated function are given by roots of the polynomial

$$
1+\sum_{k=0}^{N-2} z^{2^{k}}-z^{2^{N-1}}=0
$$

This is also just a lacunary polynomial. In Figure 4 the numerical result of the Padé approximation for $f_{\mathrm{Jac}}(z)$ is shown. The poles and zeros are plotted for the [64 | 64] Padé approximation in Figure 4(a). Inside the circle $|z|=1$ some cancellations of the ghost pairs appear. The poles and zeros accumulate around $|z|=1$ with the increase of order of the Padé approximation. In the case of the $M=64$, the poles accumulate around $|z|=1$ with making the zero-pole pairings. Figure 4(b) shows the Padé approximated functions in the polar-form with $r=1$. It well approximates the original function $f_{\mathrm{Jac}}(z)$ when the order of the Padé approximation increases.

It is also shown that the complex zeros of the polynomial (17) cluster near unit circle $|z|=1$ and distribute uniformly on the circle as $F_{N} \rightarrow \infty$ by Erdos-Turan-type theorem given in Appendices C and B [34-41].
4.2. Example 2: Fibonacci Lacunary Series. As a second example, we would like to apply Padé approximation to the following lacunary series:

$$
f_{\mathrm{Fib}}(z)=\sum_{n=0}^{\infty} z^{F_{n}}
$$

where $F_{n}$ is $n$th Fibonacci number. This function also has a natural boundary on $|z|=1$. The Padé approximated function exactly has the following form:

$$
\begin{aligned}
f_{\mathrm{Fib}}^{\left[F_{N}\right]}(z) & \sim f_{\mathrm{Fib}}^{\left[F_{N} / 2 \mid F_{N} / 2\right]}(z) \\
& =\frac{A_{\mathrm{Fib}}^{F_{N}}(z)}{1+z^{F_{N-4}}-z^{F_{N-2}}} .
\end{aligned}
$$

The explicit form of the numerator $A_{\mathrm{Fib}}^{F_{N}}(z)$ is given in Appendix D. The poles of the $\left[F_{N} / 2 \mid F_{N} / 2\right]$ Padé approximated function are given by zeros of the lacunary polynomial

$$
1+z^{F_{N-4}}-z^{F_{N-2}}=0 .
$$

In Figure 5 the numerical result of the Padé approximation to $f_{\mathrm{Fib}}(z)$ is shown. The poles and zeros are plotted for the [55 | 55] Padé approximation in Figure 5(a). The poles and zeros accumulate around $|z|=1$ with the increase of the order of the Padé approximation. No pole appears inside the unit circle. The original function is also well approximated by the [56 56] Padé approximation (see Figure 5(b)). 


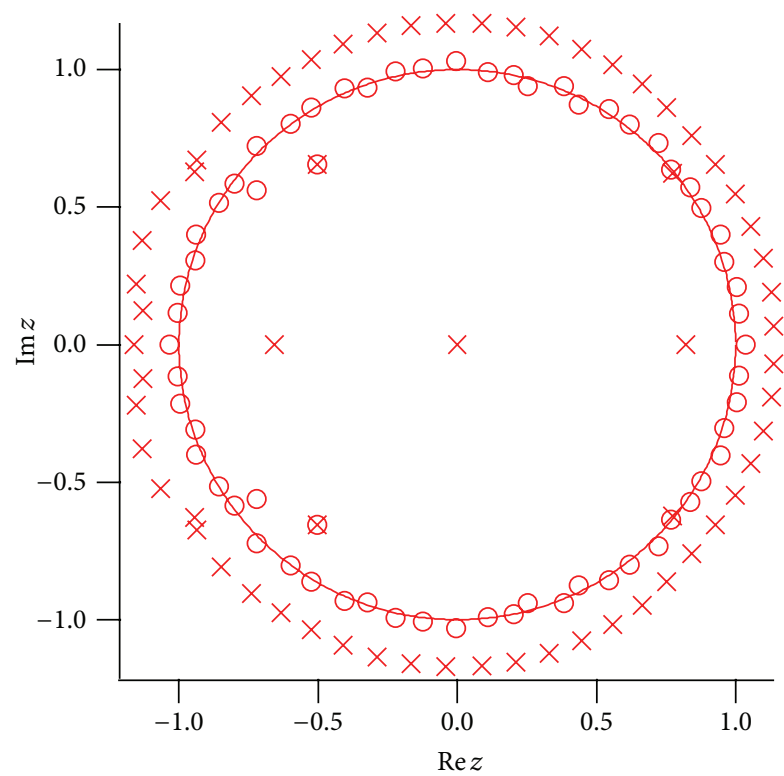

(a)

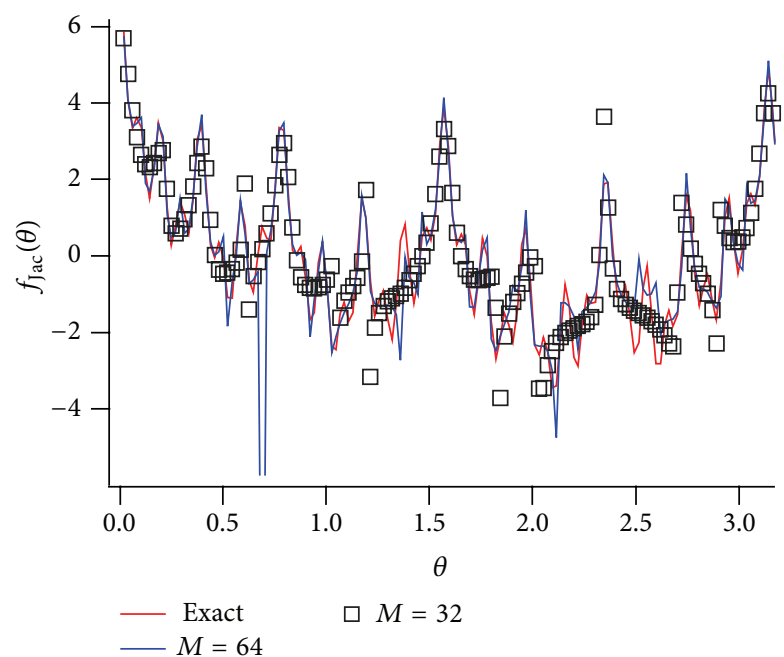

(b)

Figure 4: (a) Distribution of poles $(\bigcirc)$ and zeros $(x)$ of the [64 | 64] Padé approximation for the test function $f_{\mathrm{Jac}}(z)$ with a natural boundary on $|z|=1$. The unit circle is drawn to guide the eye. (b) The Padé approximated functions $f_{\mathrm{Jac}}^{[32 \mid 32]}(\theta), f_{\mathrm{Jac}}^{[64 \mid 64]}(\theta)$ and the exact function $f_{\mathrm{Jac}}(\theta)$ in the polar-form with $r=1.0$ (after [32]).

\section{Natural Boundary of Random Power Series and the Noise Effect on Padé Approximation}

In this section, we apply Padé approximation to the random power series with a natural boundary with probability 1 and investigate how the approximation detect the singularity of the series. In addition, we examine the effect of noise on the coefficients of the power expansion for some test functions. Some related theorems for the natural boundary of the function generated by the random power series are given in Appendix F.

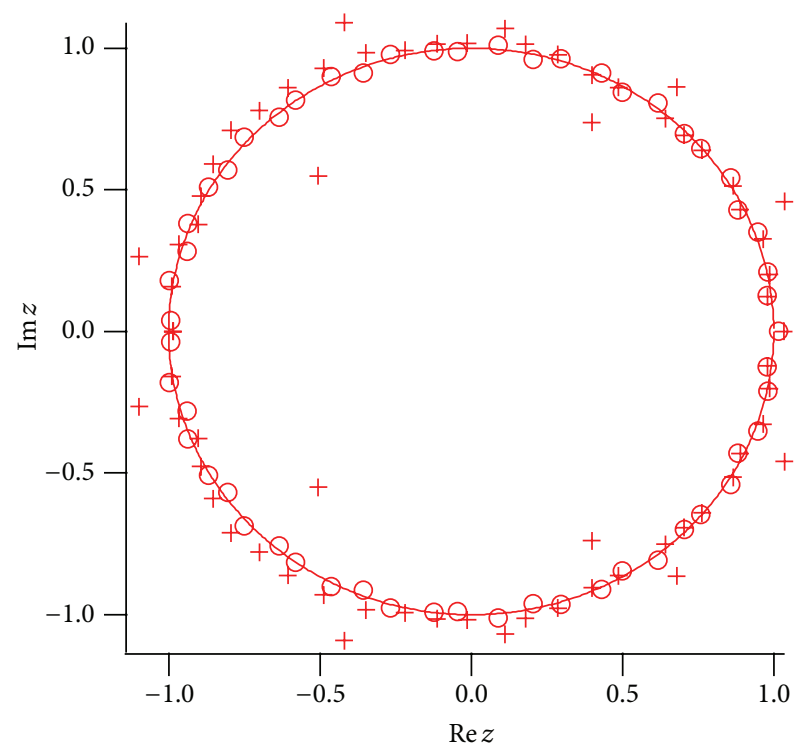

(a)

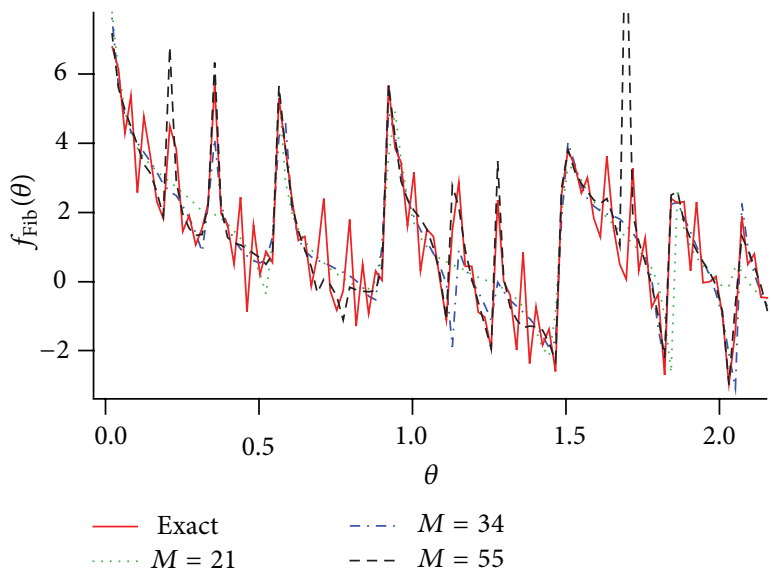

(b)

FIgure 5: (a) Distribution of poles $(\bigcirc)$ and zeros $(+)$ of the [55 | 55] Padé approximated function for the test function $f_{\mathrm{Fib}}(z)$ with a natural boundary on $|z|=1$. The unit circle is drawn to guide the eye. (b) The Padé approximated functions $f_{\mathrm{Fib}}^{[21 \mid 21]}(\theta), f_{\mathrm{Fib}}^{[34 \mid 34]}(\theta)$, and $f_{\mathrm{Fib}}^{[55 \mid 55]}(\theta)$ corresponding to $N=F_{9}=55, N=F_{10}=89$, and $N=F_{11}=144$, respectively, and the exact function $f_{\mathrm{Fib}}(\theta)$ in the polar form with $r=1.0$ (after [32]).

5.1. Random Power Series and Natural Boundary. Let us consider a random power series:

$$
f_{\text {noisel }}(z)=\sum_{n=0}^{\infty} \epsilon r_{n} z^{n} .
$$

Here the coefficients, $r_{0}, r_{1}, r_{2}, \ldots$, are i.i.d. random variables which take a value within $r_{n} \in[0,1]$, and $\epsilon$ is the strength of the randomness. It is shown that, in general, the random power series has a natural boundary on the unit circle $|z|=1$ with probability one. Figure 6(a) shows distribution of poles and zeros of the [50 | 50] Padé approximated function for 


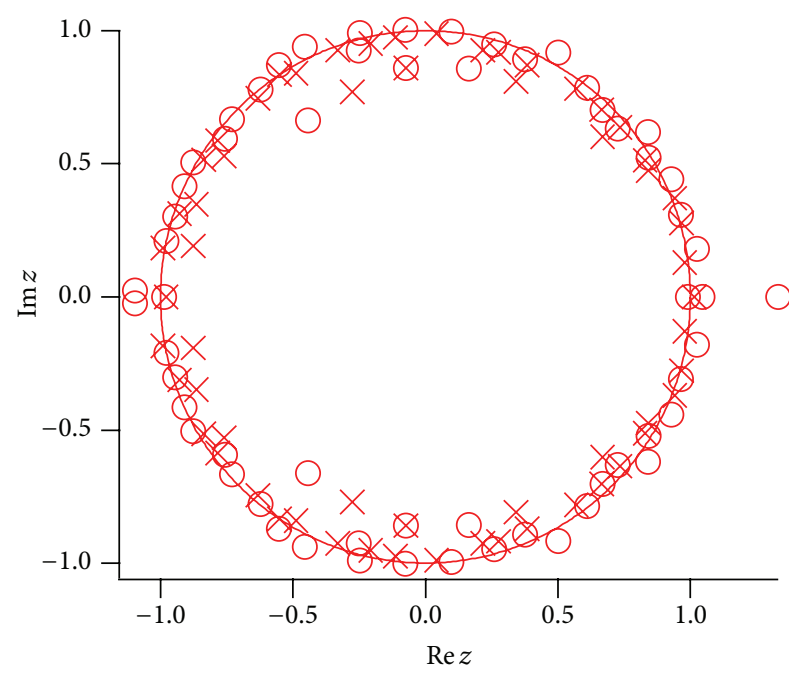

(a)

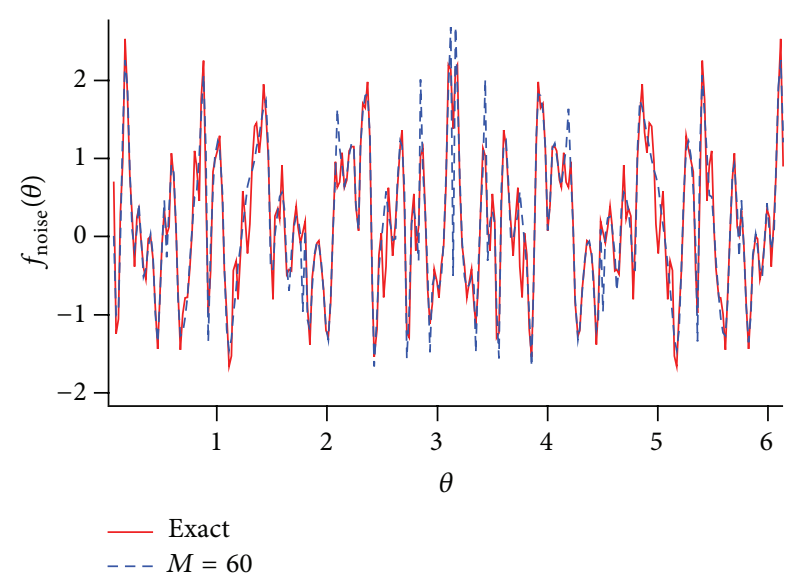

(b)

Figure 6: (a) Distribution of poles $(\bigcirc)$ and zeros $(x)$ of the Padé approximated function $f_{\text {noisel }}^{[50 \mid 50]}(z)$ for a random power series $f_{\text {noise } 1}(z)$ with $\epsilon=1$. The unit circle is drawn to guide the eye. (b) The Padé approximated function $f_{\text {noisel }}^{[50 \mid 50]}(\theta)$ and the exact function $f_{\mathrm{Fib}}(\theta)$ in the polar form with $r=1.0$.

$f_{\text {noisel }}(z)$. Some pairs of poles and zeros are perfectly cancelled inside the circle $|z|=1$. On the other hand, almost all the poles and zeros of the Pade approximated function assemble around the circle $|z|=1$ and not cancelled. The pair of poles and zeros around the circle $|z|=1$ is called "Froissart doublets," and it well corresponds to the natural boundary of $f_{\text {noise } 1}(z)$. The original function is also well approximated by the $[50 \mid 50]$ Padé approximation (see Figure 6(b)).

Figure 7 shows an example of the coefficients $\left\{c_{n}\right\}=\left\{\epsilon r_{n}\right\}$ of the random power series and the coefficients $\left\{a_{n}\right\}$ and $\left\{b_{n}\right\}$ of the [50 | 50] Padé approximated function. The fluctuation of the coefficient $\left\{b_{n}\right\}$ that determines the poles of the Padé approximated function is smaller than that $\left\{a_{n}\right\}$ of the numerator.

Note that the truncated random series is a random polynomial. As for the random polynomial, it is well known that the distribution of the zeros converges on the uni circle when

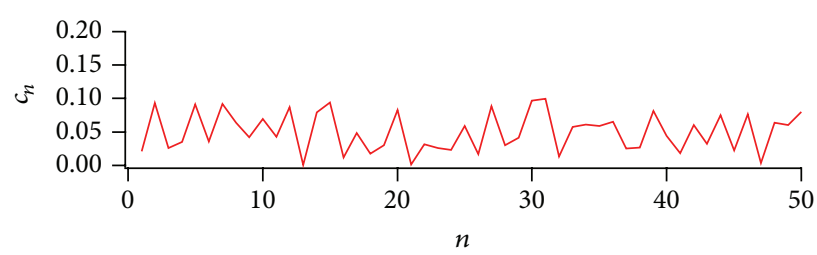

(a)

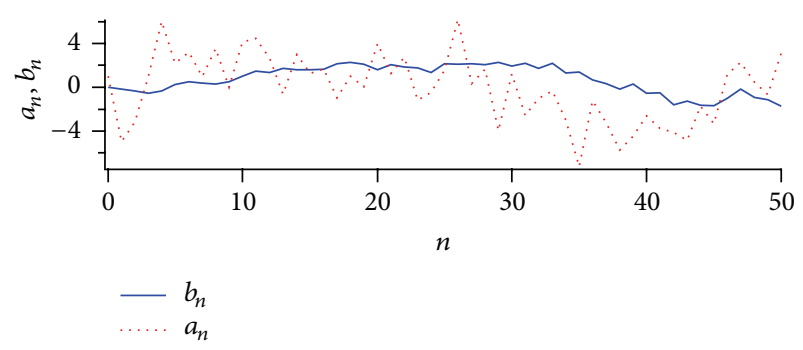

(b)

FIgURE 7: (a) The coefficient $\left\{c_{n}\right\}=\left\{\epsilon r_{n}\right\}$ of a truncated random power series $f_{\text {noisel }}^{[100]}(z)$ with $\epsilon=0.1$. (b) The coefficients $\left\{a_{n}\right\}$ and $\left\{b_{n}\right\}$ of the Padé approximated function $f_{\text {noisel }}^{[50 \mid 50]}(z)$ for $f_{\text {noisel }}^{[100]}(z)$.

the order of the random polynomial increases (Erdos-Turantype theorem) $[34,35,40]$. Accordingly, we can generally interpret that in the Padé approximated function to the random power series the distribution of poles and zeros also accumulates around the unit circle when the order of the Padé approximation increases. The dependence of the zeros of the random polynomial and the zeros and poles of the Padé approximation has been studied by Gilewicz and Kryakin [42] and Ding and Xiao [43].

5.2. Effect of Noise on a Function with a Simple Pole. In the following subsections, we investigate influences of noise on the Padé approximation for some constructed noisy test functions as follows;

$$
f_{\text {test+noise2 }}(z)=f_{\text {test }}(z)+f_{\text {noise2 }}(z) \text {, }
$$

where $f_{\text {test }}(z)=\sum_{n=0}^{\infty} a_{n} z^{n}$ and $f_{\text {noise2 }}(z)=\sum_{n=0}^{\infty} \epsilon_{n} z^{n}$. The $\left\{\epsilon_{n}\right\}$ is i.i.d. random variables within $[-\epsilon, \epsilon]$, where $\epsilon$ is the noise strength. Essentially, $f_{\text {noise2 }}(z)$ is the same as the random power series $f_{\text {noisel }}(z)$. First of all, in this subsection, we consider a truncated function with a simple pole. Note that if $a_{n}=C$ (constant) and $\epsilon=0$, that is, in noise-free case, $f_{\text {pole+noise }}(z)=C \sum_{n=0}^{\infty} z^{n}=C /(1-z)$ with a simple pole at $z=1$. In [2] by Baker Jr., the noise effect is summarized as follows: the $[M \mid M]$ Padé approximation has an unstable zero at the distance of order $\epsilon^{-1}$ from the origin, and the other zeros make $(M-1)$ Froissart doublets (zero-pole pairs).

Next, we consider a function

$$
\begin{aligned}
f_{\text {pole2 }+ \text { noise } 2}(z) & =f_{\text {pole2 }}(z)+f_{\text {noise } 2}(z), \\
& =\sum_{n=0}^{\infty}\left(\frac{1}{2^{n}}+\epsilon_{n}\right) z^{n}
\end{aligned}
$$




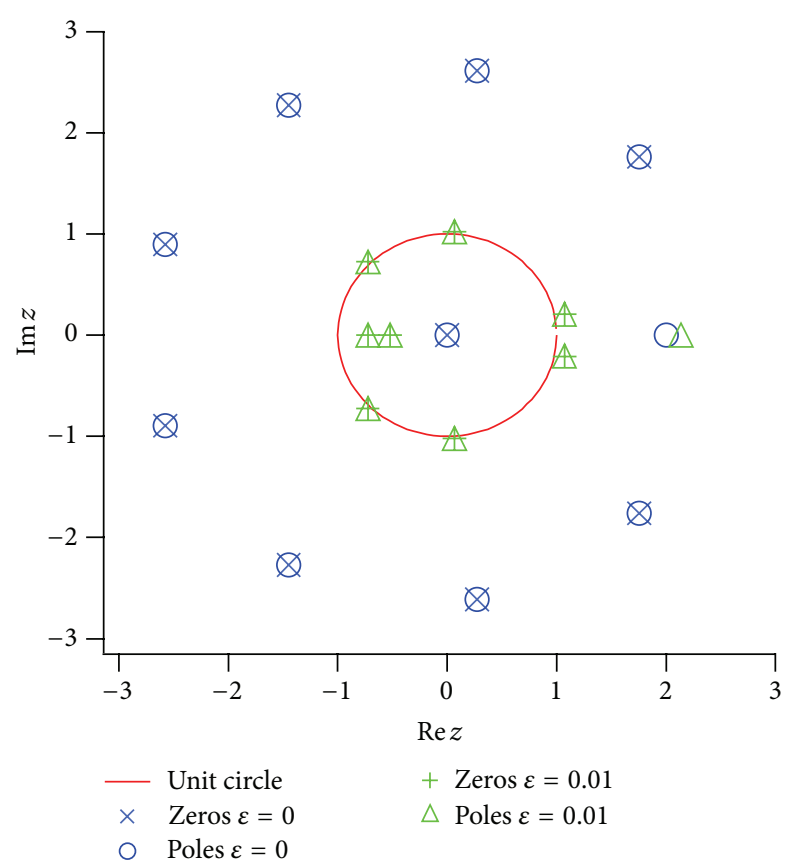

FIGURE 8: Distribution of poles $(\bigcirc, \triangle)$ and zeros $(\times,+)$ of the $[10 \mid$ 10] Padé approximated function $f_{\text {pole2+noise } 2}^{[10 \mid 10]}(z)$ with a stable pole at $z=2$ for noise strength $\epsilon=0, \epsilon=0.01$. The unit circle is drawn to guide the eye.

with the noise strength $\epsilon<1$. Note that

$$
f_{\text {pole2 }}(z)=\frac{2}{(2-z)}
$$

with a simple pole at $z=2$ to clearly show the shift of the poles of the approximated function due to the noisy series.

Figure 8 shows distribution of the poles and zeros of the [10 | 10] Padé approximated functions. It clearly shows the pole shift by the noise effect. In the noise-free case $(\epsilon=0)$, a pole of the Padé approximation appears at $z=2$ and the other poles are cancelled with zeros (zero-pole ghost pairs). In a case when the relatively small noise $(\epsilon=0.01)$ is added, the poles and zeros move toward $|z|=1$ with making Froissart doublets, although a pole at $z=2$ is quite stable. It becomes impossible to detect the true pole at $z=2$ when the noise strength is relatively large $(\epsilon=0.1)$, not shown in Figure 8 .

As a result, it is found that the locations of the ghost pairs are unstable for noise, and the residues for the poles are much smaller than one corresponding to the true pole. We can guess that the proximity of the nonmodal poles and zeros of the Padé approximated function can be understood in a sense that the poles due to the noise need zeros to cancel with each other as $\epsilon \rightarrow 0$.

5.3. Effect of Noise on a Function with a Branch Cut. We investigate the effect of the noise on functions with a branch cut. First, let us consider a function

$$
f_{\text {branch } 1}(z)=\sqrt{\frac{3+z}{1+z}}
$$

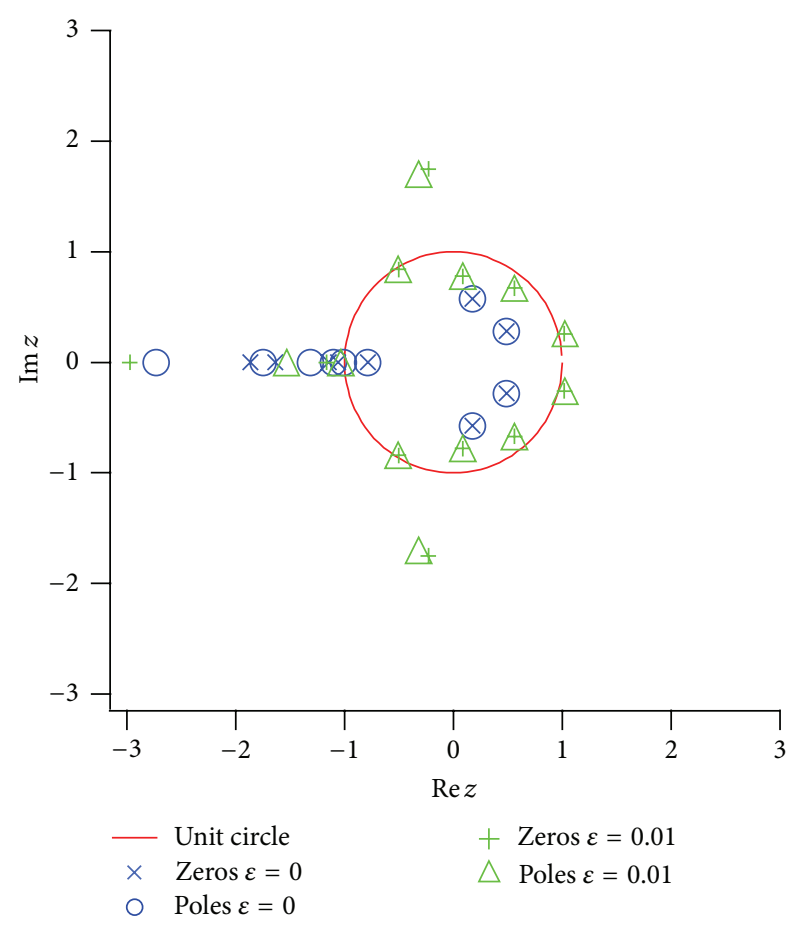

FIGURE 9: Distribution of poles $(\bigcirc, \triangle)$ and zeros $(\times,+)$ of the $[10 \mid$ 10] Padé approximated function $f_{\text {branchl+noise } 2}^{[10 \mid 1}(z)$ with a brunch cut from $z=-\infty$ to $z=0$ for the noise strength $\epsilon=0, \epsilon=0.01$. The unit circle is drawn to guide the eye.

with an algebraic branch points at $z=-1$ and $z=-3$ and with the branch cut in $[-3,-1]$. Distribution of the poles and zeros of the Padé approximated function $f_{\text {branch1+noise2 }}^{[10 \mid 10]}(z)$ is shown in Figure 9. In a case with relatively small noise $(\epsilon=0.01)$, some poles make a line on the branch cut, and some poles and zeros move toward the unit circle $|z|=1$. It is impossible to detect the branch cut when the noise strength is relatively large $(\epsilon=0.1)$.

Next, let us consider a function

$$
f_{\text {branch2 }}(z)=\log \left(\frac{6}{5}-z\right)
$$

with a logarithmic branch point at $z=6 / 5$ and with a brunch cut from $z=6 / 5$ to $z=\infty$. The distribution of the poles and zeros of the Padé approximated function $f_{\text {branch2+noise2 }}^{[10 \mid 10]}(z)$ for the $f_{\text {branch2 }}(z)$ with the noisy perturbation is shown in Figure 10. Some poles and zeros are making a line alternatively on the branch cut in the noise-free case $(\epsilon=0)$. It assembles around the unit circle $|z|=1$ with making Froissart doublets when the noise with strength $\epsilon=0.01$ is added.

5.4. Effect of Noise on a Function with a Natural Boundary. Figure 11 shows distribution of the poles and zeros of the [50 | 50] Padé approximated function for

$$
f_{\mathrm{Jac}+\text { noise }}(z)=f_{\mathrm{Jac}}(z)+f_{\text {noise2 }}(z),
$$

which has a natural boundary on $|z|=1$. 


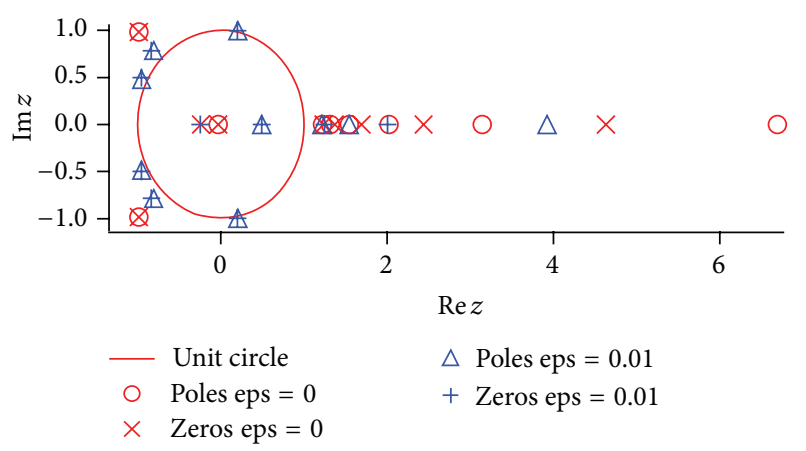

Figure 10: Distribution of poles $(\bigcirc, \triangle)$ and zeros $(\times,+)$ of the $[10 \mid$ 10] Padé approximated function $f_{\text {branch } 2+\text { noise } 2}^{[10 \mid 10]}(z)$ with a brunch cut from $z=6 / 5$ to $z=\infty$ for the noise strength $\epsilon=0, \epsilon=0.01$. The unit circle is drawn to guide the eye.

In the noise-free case, the pairs of poles and zeros of the Padé approximated function are perfectly cancelled inside the unit circle $|z|=1$. The other poles and zeros of the Padé approximated function assemble around the circle $|z|=1$ without cancellation. In the relatively small noise case $(\epsilon=$ 0.01 ), the location of the poles is not significantly changed compared with the zeros shifted outside the unit circle due to the noise effect. And, again, the poles and zeros move toward $|z|=1$ with making Froissart doublets when the noise strength is relatively large $(\epsilon=0.1)$. It is closely related to a fact that fluctuation of the coefficients of the numerator of the Padé approximated function is much larger than those in the denominator, as seen in Padé approximation to the random power series in Figure 7. As a result, the singularity of the Padé approximated function for the function with a natural boundary is more sensitive to the noisy perturbation than that in the functions with the other type singularity such as simple poles and branch points.

It is very difficult to effectively distinguish whether the poles of the Padé approximation originated from the natural boundary on $|z|=1$ of the original function $f_{\mathrm{Jac}}(z)$ or from the other natural boundary on $|z|=1$ generated by noisy series $f_{\text {noise2 }}(z)$ or numerical errors. Actually the round-off error affects the distribution of the poles and zeros of the Padé approximated function. Accordingly, to determine the expansion coefficients $c_{n}$ with adequate accuracy becomes very important in the numerical calculation. This is a drawback of the Padé approximation when we use it for functions with unknown singularities.

5.5. Numerical Accuracy and Spurious Poles. As we observed in the last subsection, the effect of rounding error and accuracy limit of computers work in the numerical results of the Padé approximation. As the result of accumulation of the round-off error, the "spurious poles" appear around the unit circle $|z|=1$ as the pole-zero pairs when the order of Padé approximation increases (we used a term "Froissart doublets" for the poles-zero pairs generated by random power series, conveniently, although we cannot numerically distinguish it from the spurious poles due to the round-off errors; in the next section, we will discuss the Froissart doublets again).

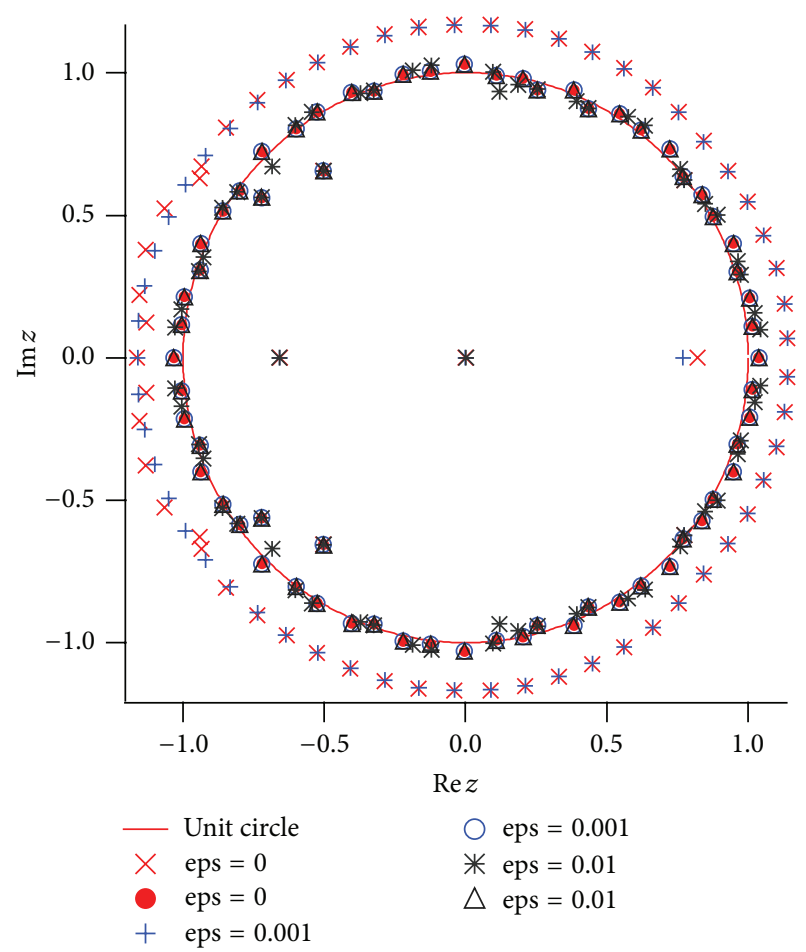

FIgURE 11: Distribution of poles $(\bullet, \bigcirc, \triangle)$ and zeros $(\times+, *)$ of the Padé approximated function $f_{\text {Jac+noise }}^{[50 \mid 50]}(z)$ for the lacunary series $f_{\text {Jac+noise }}^{[100]}(z)$ with a natural boundary on $|z|=1$. The noise strengths are $\epsilon=0, \epsilon=0.001$, and $\epsilon=0.01$, respectively. The unit circle is drawn to guide the eye.

However, we can roughly distinguish between true poles and the spurious poles by "residue analysis" of the Padé approximated function because the spurious poles-zero pairs are unstable for the change of the order. In this subsection, we try to investigate the residues of the Pade approximation for some test functions. Up to now, the residue analysis has been mainly used for performance comparison between the different algorithms of the Padé approximation of the same order $[30,31]$. On the other hand, it seems that the study by using the information of the residue analysis is still rare in the Padé approximation $[10,12]$.

Generally, the rational polynomials of the diagonal Padé approximation can be uniquely identified by the poles $\left\{z_{k}\right\}$ and the corresponding residues $A_{k}$ as follows:

$$
\frac{Q_{M}(z)}{P_{M}(z)}=\sum_{k}^{M} \frac{A_{k}}{z-z_{k}},
$$

where the residues are given by

$$
A_{k}=\frac{Q_{M}\left(z_{k}\right)}{\prod_{j(\neq k)}^{M}\left(z_{k}-z_{j}\right)} .
$$

Here, we investigate the convergence property of the magnitude of residues $\left|A_{k}\right|$ arranged in descending order.

Figure 12 shows the absolute value of the residues $\left|A_{k}\right|$ of some Padé approximated functions for the test function 


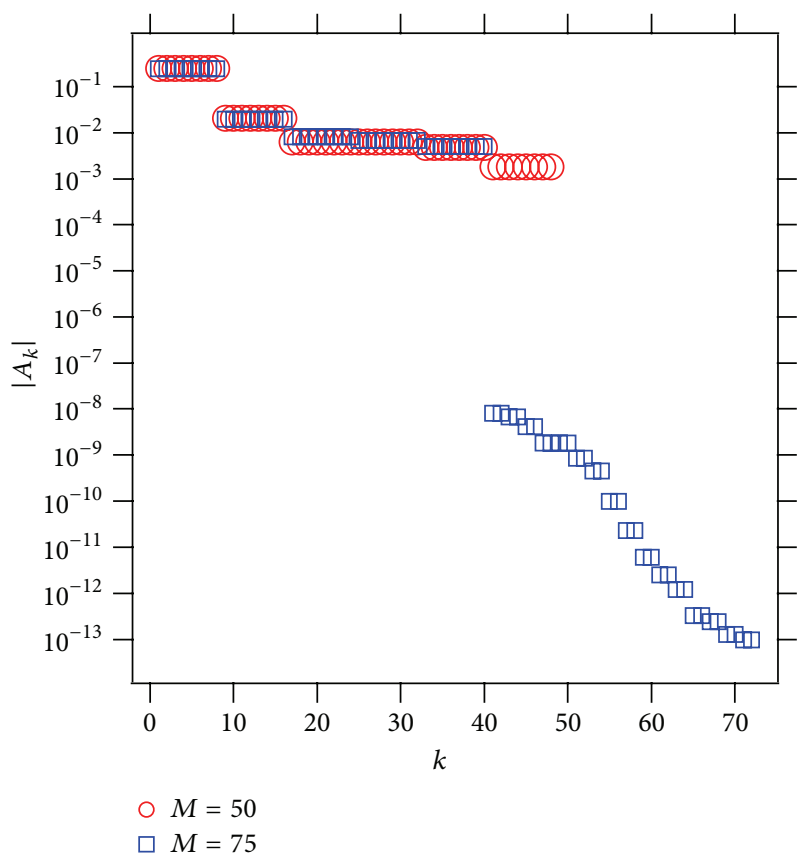

FIGURE 12: Absolute values of the residues $\left|A_{k}\right|$ of the [50 $\left.\mid 50\right]$ and [75 $\mid 75]$ Padé approximated functions for the test function $f_{5}(z)$ without noise. The $\left|A_{k}\right|$ are arranged in descending order.

$f_{5}(z)$, which are arranged in descending order (note that they are noise-free cases.) The distribution of the poles and zeros of the Padé approximated functions is given in Figure 3. In a case of $M=50$, the magnitude of the all residues $\left|A_{k}\right|$ is larger than $O\left(10^{-3}\right)$, which correspond to the relevant poles arranged radially in eight directions from the true poles. On the other hand, in a case of $M=75$, the spurious poles appear and distribute around the unit circle $|z|=1$ (see Figure 3(b)). It is found that the absolute values of the residues corresponding the spurious poles are several order of magnitude smaller than the relevant poles.

Distribution of poles and zeros of the Padé approximated function $f_{\text {branch2 }}^{[20 \mid 20]}(z)$ for the test function $f_{\text {branch2 } 2}(z)$ is shown in Figure 13. The stable poles and zeros are lined on $[6 / 5, \infty]$, and the spurious poles appear around $|z|=1$. The magnitude of the residues of the spurious poles is also enormously small compared with that of the stable poles remaining with the increase of the order of the Padé approximation.

Figure 14 is also the result of the residues analysis for the Padé approximated function for the test function $f_{\text {Jac }}(z)$ with a natural boundary on the unit circle $|z|=1$. In the [50 | 50] Padé approximated function, the magnitude of the residues $\left|A_{k}\right|$ is shown in changing the noise strengths $\epsilon=0,0.01,0.1$ corresponding to poles-zeros distribution in Figure 11.

In the small noise case $(\epsilon=0.01)$, the results of the residue analysis for $f_{\mathrm{Jac}+\text { noise2 }}(z)$ is almost the same as the noise-free case $(\epsilon=0)$, and in the case with relatively strong noise $(\epsilon=$ 0.1 ), the noise shifts the magnitude of the residues with larger value. In addition, the result of the residue analysis of the noise-free cases for some different orders of the Padé approximation is shown in Figure 14(b). We should have in mind that

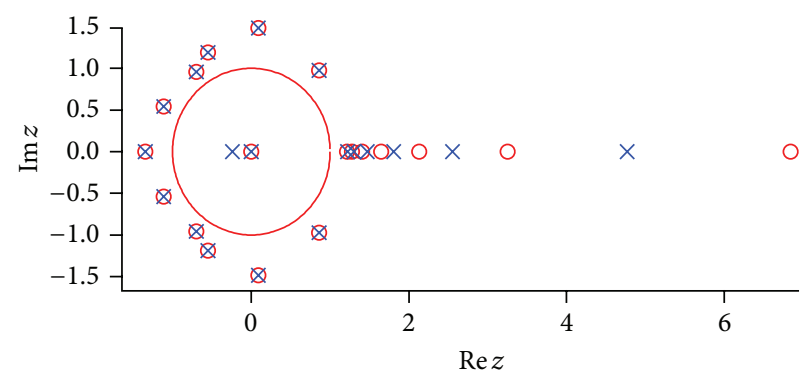

(a)

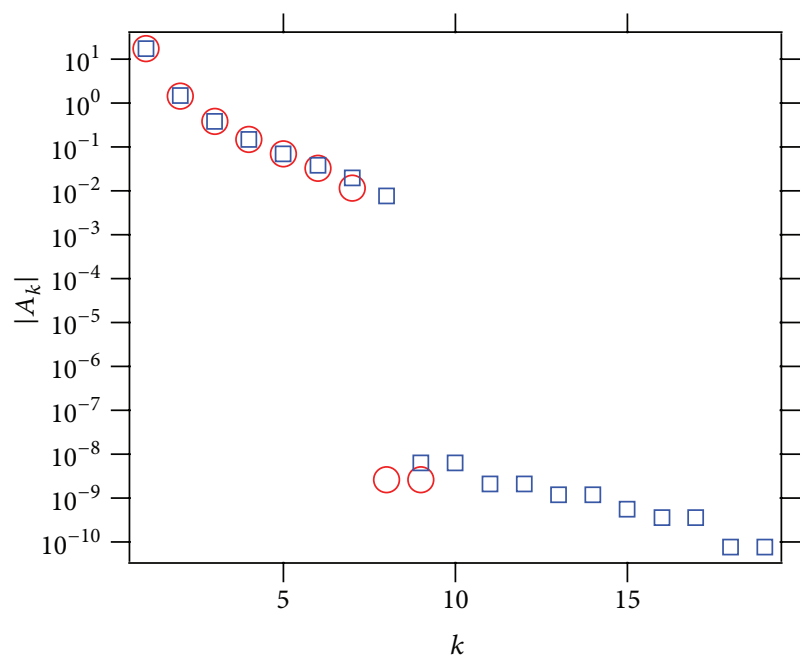

○ $M=10$

$\square M=20$

(b)

Figure 13: (a) Distribution of poles $(\bigcirc)$ and zeros $(\times)$ of the [20 | 20] Padé approximated function $f_{\text {branch } 2}^{[20 \mid 20]}(z)$. The unit circle is drawn to guide the eye. (b) Absolute values of the residues $\left|A_{k}\right|$ of the [10 $\mid$ 10] and [20|20] Padé approximated functions for the test function $f_{\text {branch2 }}(z)$ without noise. The residues are arranged in descending order.

the order is important when we apply Padé approximation to the lacunary power series because we should not take the order of the approximation in the gap of the series.

\section{Froissart Doublets}

The problem of constructing the $Z$-transform $Z(z)$ of a finite time-series is a standard problem in mathematics [1014]. For example, it is shown that for a sum of oscillating damped signals, the $Z$-transform associated with the timeseries can be characterized by a sum of the poles of the Padé approximated function. The position of each pole is simply linked to the damping factor and the frequency of each of the oscillators. Also, it is important to note that all these poles lie strictly outside the unit circle because it corresponds to the damping [10-13]. In addition, we will consider quasianalyticity property of the random power series by the residue analysis of the Padé approximation. 


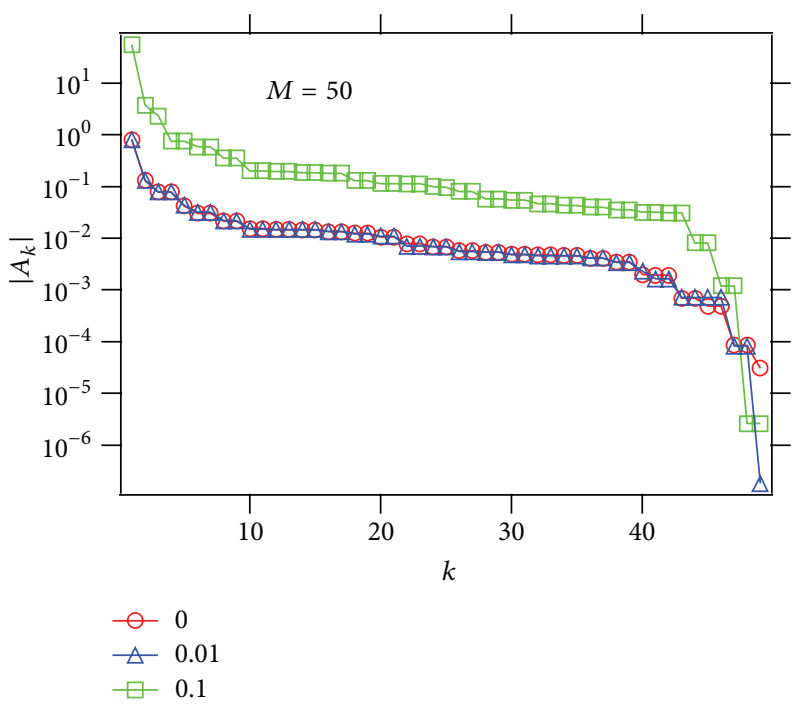

(a)

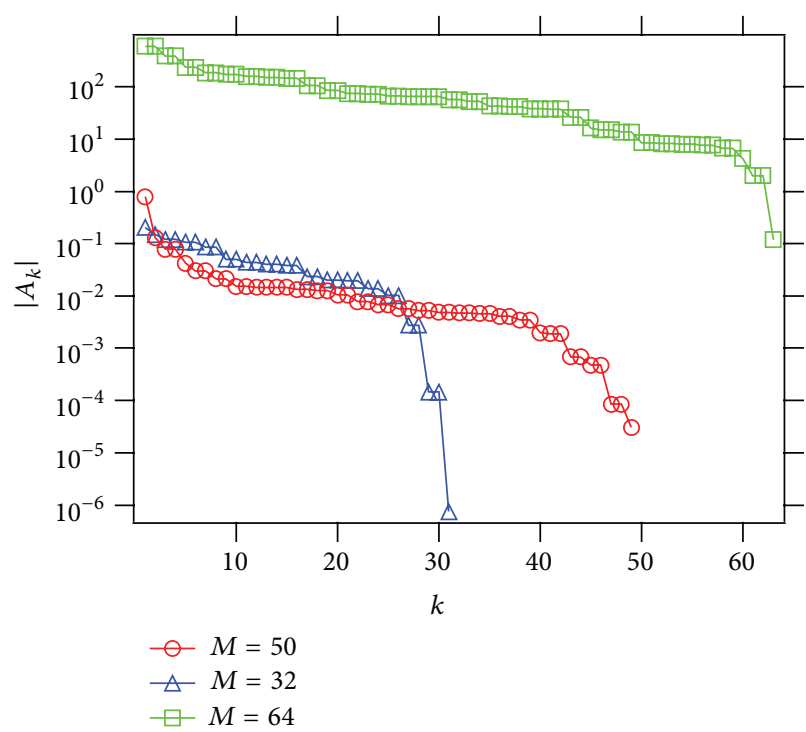

(b)

Figure 14: (a) Absolute values of the residues $\left|A_{k}\right|$ of the [50 50] Padé approximated functions to the noise added test function $f_{\text {Jac+noise }}(z)$ with the strength $\epsilon=0,0.01,0.1$. (b) Absolute values of the residues $\left|A_{k}\right|$ of the Padé approximated functions $f_{\text {Jac }}^{[50 \mid 50]}(z), f_{\text {Jac }}^{[32 \mid 32]}(z)$, and $f_{\mathrm{Jac}}^{[64 \mid 64]}(z)$ for the test function $f_{\mathrm{Jac}}(z)$ without the noise. The residues are arranged in descending order.

6.1. Noise Attractor. In signal processing, we can use the fact that the poles and zeros of the Padé approximated function to the noisy series distribute around the unit circle $|z|=1$ when we remove the noise from the observed data through $Z$-transform and/or Fourier transform of the data. Let a sequence $\left\{s_{0}, s_{1}, \ldots, s_{n}, \ldots\right\}$ be a sample signal without noise. Then we define the $Z$-transform of the sequence as

$$
Z(z)=\sum_{n=0}^{N} s_{n} z^{n} .
$$

The function $Z(z)$ is analytic interior of $|z|<1$ if the number of signals $N$ is finite [44]. Note that discrete Fourier transform is a special case of the $Z$-transform.

Next, let us consider a signal sequence in $t \in[0, T]$ consisting of the superimposed damping oscillators as

$$
s_{k}=\sum_{\ell} A_{\ell} e^{i \omega_{\ell}(k / N) T}, \quad k=0,1, \ldots, N-1,
$$

where $A_{\ell}$ is the amplitude of the $\ell$ th oscillator and $\omega_{\ell}=$ $2 \pi f_{\ell}+i \alpha_{\ell}$. Here, $f_{\ell}$ and $\alpha_{\ell}$ are the frequency and the damping factor of the $\ell$ th oscillator. Then, the $Z$-transform is

$$
\begin{aligned}
Z(z) & =\sum_{n=0}^{\infty} s_{n} z^{n} \\
& =\sum_{n=0}^{\infty} \sum_{\ell} A_{\ell} e^{i \omega_{\ell}(n / N) T} z^{n} \\
& =\sum_{\ell} \frac{A_{\ell}}{1-z z_{\ell}},
\end{aligned}
$$

where we take a limit $n \rightarrow \infty$ keeping $T / N$ and $z_{\ell} \equiv e^{i \omega_{\ell}(T / N)}$. Accordingly, the singularity of $Z(z)$ appears as the poles at $z=z_{\ell}^{-1} \equiv e^{-i \omega_{\ell}(T / N)}$ outside the unit circle $|z|>1$, and the residue is $\operatorname{Re} s\left(z_{\ell}^{-1}\right)=z_{\ell}^{-1} A_{\ell}$.

On the other hand, let us consider a noise-added sequence $\left\{S_{0}, S_{1}, \ldots, S_{n}, \ldots\right\}$. Then, the Froissart pointed out that there are two types of the poles: stable poles and unstable poles when we apply the diagonal Padé approximation to the unknown data set. In general, the $Z$-transform $Z(z)=$ $\sum_{n=0}^{N} S_{n} z^{n}$ of the noisy sequence has a natural boundary on the unit circle $|z|=1$ with probability 1 . In fact, the poles and zeros (Froissart doublets) of the Padé approximated function often distribute around the unit circle when the numerical error and/or noise are mixed into the Taylor series of the analytic functions, as seen in the last section. That is to say, we sometimes call the unit circle $|z|=1$ noise attractor in a sense that the poles and zeros are attracted to the circle as the Froissart doublets [45]. Accordingly, it is found that Padé approximated function for the function $Z(z)$ has stable poles associated with the damping modes and unstable spurious poles associated with the noisy fluctuation. After elimination of the spurious poles around the noise attractor from the noisy sequence we can reconstruct the noise-free sequence consisting of the stable poles located in the domain $|z|>1$. Another remarkable feature of the nonmodal poles is that the absolute values of the Cauchy residues associated with them are usually much smaller than those associated with true poles.

6.2. Random Power Series and Quasianalytic Function. Weierstrass defined the analytic function by direct analytic continuation of function. Then, apparently the analytic continuation is impossible beyond the natural boundary even if we 
can uniquely define the function and it is analytic outside the analytic domain. Borel and Gammel extended the narrow condition for the analyticity and gave a definition of quasianalytic functions [46, 47]. Gammel conjectured the following for the random power series $[10,45]$.

Gammel Conjecture (1973). The random power series belongs to the Borel class of quasianalytic functions as the following form:

$$
f_{\text {Gammel }}(z)=\sum_{k=0}^{\infty} \frac{B_{k}}{1-w_{k} z},
$$

where $w_{k}=e^{i 2 \pi X_{k}}$ and $\left\{X_{k}\right\}$ are real numbers in the interval in $X_{k} \in[0,1]$ and $B_{k}$ decreases rapidly with $k$. Then, the natural boundary in the Weierstrass sense can be crossed.

The function (33) is a simple example that poles are densely distributed on the unit circle. Then the convergence property of the sequence $\left|B_{k}\right|$ is important for the analyticity of the function. Carleman proved that $f_{\text {Gammel }}(z)$ is quasianalytic if $B_{k}$ satisfies the following condition:

$$
\left|B_{k}\right|<C e^{-k^{1+c}}, \quad c>0 .
$$

This is Carleman class of quasianalytic functions. See Gammel's paper [45] for the details. Moreover, Gammel and Nuttall proved that the quasianalytic functions can be exactly approximated by the Padé approximation [45].

Gammel-Nuttall Theorem (1973). If $B_{k}$ in (33) satisfies the condition (34) and $\left|\omega_{k}\right|=1$, then the sequence of $[N+J \mid N]$ Padé approximation to the $f_{\text {Gammel }}(z)$ converges in measure to the function $f_{\text {Gammel }}(z)$ as $N \rightarrow \infty$ in any closed, bounded region of the complex plane, where $J$ is a natural number that equals $N$ or less.

Is the Gammel Conjecture True? We try to examine the validity of the Gammel conjecture by applying residue analysis of the Padé approximated function to the random power series $f_{\text {noise2 }}(z)$. Figure 15 shows the absolute values of the residues $\left|A_{k}\right|$ of the Padé approximated functions $f_{\text {noise }}^{[45 \mid 45]}(z)$ for three different samples in descending order. $\left|A_{k}\right|$ roughly exponentially decreases with respect to $k$ as

$$
\left|A_{k}\right| \sim \exp (-\beta k)
$$

where $\beta$ is the decay exponent. It shows exponential decay (or faster), and, on the surface, supports the Gammal conjecture.

However, it is not nearly so simple. We should check the stability of the exponential-like decay of the magnitude of the residues by changing the order of the Padé approximation. Figure 16 shows the result for the three different orders: $M=$ $15, M=45$, and $M=55$. It expresses an indication that the decay exponent $\beta$ does not converge to a positive certain value. It seems that the exponent behaves $\beta \rightarrow 0$ as a limit $M \rightarrow \infty$. On the other hand, if we directly apply the Padé approximation to the quasianalytic function $f_{\text {Gammel }}(z)$ with $B_{k}=e^{-k}$, the exponent $\beta$ is stable for changing the order of the Padé approximation (see Appendix E). These facts suggest that the random power series does not belong to Carleman

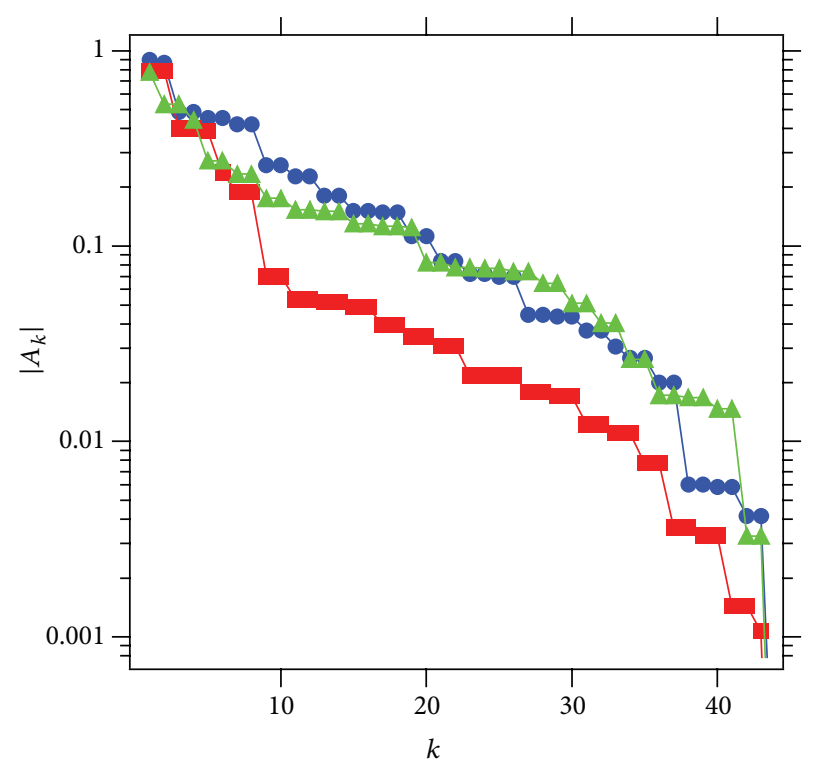

Figure 15: Absolute values of the residues $\left|A_{k}\right|$ of the Padé approximated functions $f_{\text {noise2 }}^{[45 \mid 45]}(z)$ for three samples of the truncated random power series of order $N=90$, and $\epsilon=1$. The $\left|A_{k}\right|$ are arranged in descending order.

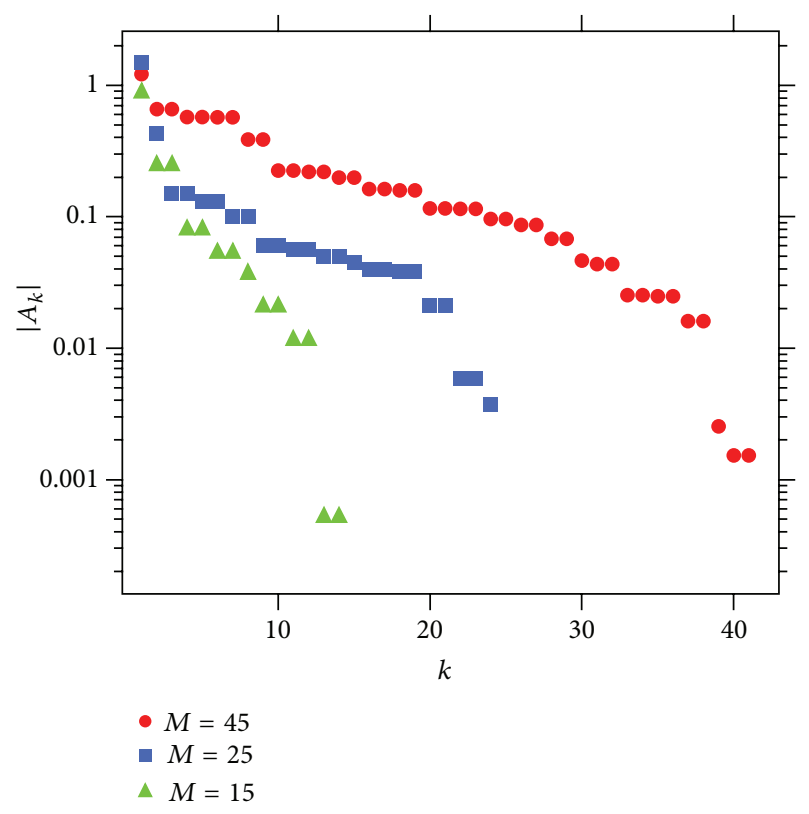

FIgUre 16: Absolute values of the residues $\left|A_{k}\right|$ of the Padé approximated functions $f_{\text {noise2 }}^{[45 \mid 45]}(z), f_{\text {noise2 }}^{[25 \mid 25]}(z)$, and $f_{\text {noise2 }}^{[15 \mid 15}(z)$ for a sample truncated random series, and $\epsilon=1$. The $\left|A_{k}\right|$ are arranged in descending order.

class of quasianalytic functions although it has a natural boundary on the unit circle, and it has the form (33). As a result, we can say that no optimism is warranted on the Gammel conjecture.

How does the residue analyses of the Padé approximation for the analyticity and/or quasianalyticity of unknown function work? It is an interesting and future problem. 


\section{Summary and Discussion}

In the present paper we numerically examined the effectiveness of the Padé approximation for some test functions with branch point, essential singularity, and natural boundary by watching the singularities of the Padé approximated functions. For the functions with a branch cut, the poles and zeros of the Padé approximated function are lined along the true branch cut. The poles and zeros are distributed around the true natural boundary if the original test function has a natural boundary. In addition, we gave the explicit Padé approximated functions for some lacunary power series which are useful to check the numerical result. It was shown that, in particular, the distribution of poles and zeros of the Padé approximated function for lacunary power series and the random power series accumulated around the unit circle when the order of the approximation increases.

We often suffer from the difficulty to distinguish whether or not the poles of the Padé approximation are intrinsically originated from the natural boundary of the original power series because the numerical errors contained in the expansion coefficients also yield a false natural boundary. Therefore, the expansion coefficients with adequate numerical accuracy are necessary when we apply the Padé approximation to functions with unknown singularities.

Furthermore, the residue calculus of the Padé approximated function is useful when we detect the singularity of the original power series from the asymptotic behavior of the truncated series. It is useful also for estimating the accuracy of the approximation. As a result, the residue calculus suggested that the random power series does not obey Gammel's conjecture; that is, it does not belong to Borel class of the quasianalytic functions.

We finally remark that the most serious problem to be improved is the numerical accuracy due to the limitation of the order in the Padé approximation when we use it for detecting unknown singularities of wave functions in quantum physics [32].

\section{Appendices}

\section{A. General Recursion Relation}

We can construct a power series that has some pole-type singularities in the following form:

$$
\frac{d z^{2}+e z+f}{a z^{2}+b z+c}=\sum_{n=0}^{\infty} a_{n} z^{n},
$$

where $a, b, c, d, e$, and $f$ are real and $c \neq 0$ for simplicity. Then the coefficients $\left\{a_{n}\right\}$ can be obtained by rearranging and comparing with the coefficients of the both sides in the same order as follows:

$$
\begin{aligned}
d z^{2}+e z+f= & \left(b a_{0} z+c a_{0}+c a_{1} z\right) \\
& +\sum_{n=2}^{\infty}\left(a a_{n-2}+b a_{n-1}+c a_{n}\right) z^{n}
\end{aligned}
$$

As a result, the power series with the pole-type singularities can be constructed by the recursion relation

$$
a_{k}=-\frac{b}{c} a_{k-1}-\frac{a}{c} a_{k-2}, \quad k \geq 2,
$$

with $c a_{0}=f, b a_{0}+c a_{1}=e$, and $a a_{0}+b a_{1}+c a_{2}=d$.

It becomes Fibonacci sequence when we set $a_{0}=0, a_{1}=$ 1 , and $a_{k}=a_{k-1}+a_{k-2}$.

\section{B. Random Polynomial}

The following theorems concerning the random power series are well known.

Erdos-Turan-Type Theorem (1950). Let us define a polynomial

$$
f(z)=\sum_{n=0}^{N} a_{n} z^{n}
$$

where coefficients $a_{n}$ are randomly distributed and $a_{0} a_{N} \neq$ 0 for simplicity. Then the zeros of the random polynomial cluster uniformly around the unit circle $|z|=1$ if "size of the truncated series" $L_{N}(f)$ is small compared to the order $N$ of the polynomial, where

$$
L_{N}(f)=\log \left(\frac{\sum_{n=0}^{N}\left|a_{n}\right|}{\sqrt{\left|a_{0} a_{N}\right|}}\right) .
$$

Note that this theorem also holds for the polynomials with deterministic coefficients $a_{n}$ such as Newman-type polynomial having coefficients in the sets $\{0,1\}$ or $\{0, \pm 1\}$.

Peres-Virag Theorem (2005). Let $\left\{a_{n}\right\}$ be i.i.d. Gaussian-type random variables; then the distribution $K(z)$ of the complex zeros $\left\{z_{k}\right\}$ of the power series

$$
f(z)=\sum_{n=0}^{\infty} a_{n} z^{n}
$$

is

$$
K\left(z_{k}\right)=\frac{1}{\pi} \frac{1}{\left(1-\left|z_{k}\right|^{2}\right)^{2}} .
$$

\section{Some Gap Theorems of Lacunary Power Series}

Weierstrass considered the analyticity of the power series

$$
f(z)=\sum_{\nu=0}^{\infty} a_{\nu} z^{b^{\nu}}, \quad b \in N, \quad b \neq 1,
$$

where $a_{v}$ is a positive number. In the main text, we set $a_{v}=$ $1, b=2$ for $f_{\text {Wei }}(z)$. Then, it is proved that the function (C.1) has a natural boundary on the unit circle $|z|=1$ if the convergence radius of the function is unity based on the following theorems for the lacunary power series. 
Hadamard-Barck Gap Theorem (1892). Let

$$
f(z)=\sum_{\nu=0}^{\infty} a_{\nu} z^{\lambda_{\nu}},
$$

where $a_{v}$ is a positive number and $\left\{\lambda_{\nu}\right\}$ denote a strictly increasing sequence of the natural numbers, satisfying an inequality $q \lambda_{v} \leq \lambda_{\nu+1}$ for $q>1$. Then the function $f(z)$ has a natural boundary on the unit circle $|z|=1$.

Fabry's Gap Theorem (1899). Power series

$$
f(z)=\sum_{\nu=0}^{\infty} a_{\nu} z^{\lambda_{\nu}}
$$

with radius of convergence $R=1$ has a natural boundary on the unit circle $|z|=1$, provided that it is Fabry series; that is,

$$
\lim _{\nu \rightarrow \infty} \frac{\lambda_{\nu}}{\nu}=\infty
$$

\section{Numerators of Diagonal Padé Approximations for $f_{\mathrm{Jac}}(z)$ and $f_{\mathrm{Fib}}(z)$}

The diagonal Padé approximation for the truncated lacunary power functions $f_{\mathrm{Jac}}(z)$ and $f_{\mathrm{Fib}}(z)$ can be exactly executed as given in the main text. The numerators $A_{\mathrm{Jac}}^{N}(z)$ and $A_{\mathrm{Fib}}^{F_{N}}(z)$ of the Padé approximated functions can be given as follows:

$$
\begin{aligned}
A_{\mathrm{Jac}}^{N}(z)= & z+2 z^{2} \\
& +2 \sum_{n=2}^{N-1} z^{H_{n}}\left(z+z^{2}+\sum_{k=1}^{n-2} z^{H_{k+2}}\right),
\end{aligned}
$$

where $H_{n}=2^{n-1}$.

Numerator of the diagonal Padé approximated function for $f_{\mathrm{Fib}}(z)$ is

$$
\begin{aligned}
A_{\mathrm{Fib}}^{F_{N}}(z)= & S_{N-4}(z) \\
& +\left[S_{N-8}(z)+z\right]\left(f_{N-4}(z)-f_{N-2}(z)\right) \\
& +\left[2 f_{N-3}(z)+2 f_{N-2}(z)+f_{N-3}(z) f_{N-6}(z)\right],
\end{aligned}
$$

where $S_{L}(z)=\sum_{k=0}^{L} f_{k}(z), f_{k}(z)=z^{F_{k}} . F_{N}$ means $N$ th Fibonacci number, and we set $F_{-1}=F_{-2}=\cdots=0$.

We have inductively obtained above results by means of Mathematica.

\section{E. Residue Analysis for Carleman Class of Quasianalytic Functions}

In this appendix, we give a direct result of residue analysis for "Carleman class" of the quasianalytic functions, for comparison with the other residue analyses in the main text. We

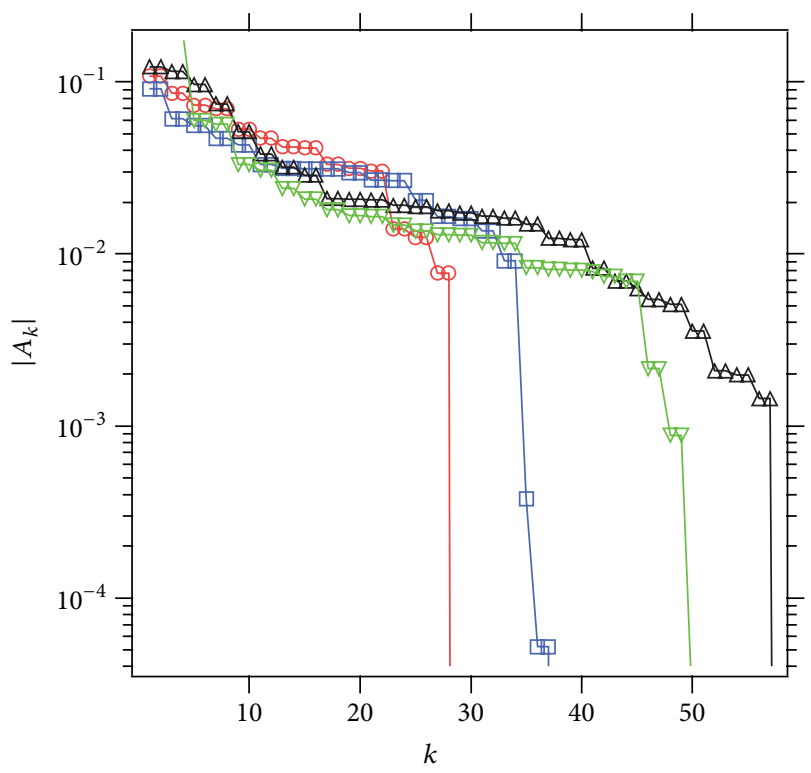

FIgure 17: Absolute values of the residues $\left|A_{k}\right|$ of the Padé approximated functions for a truncated Carleman function $f_{\text {Carleman }}(z)$ of order $M=15,25,45$, which is artificially constructed by (E.2). They are arranged in descending order in each case.

apply the Padé approximation to the quasiperiodic function $f_{\text {Carleman }}(z)$ of the Carleman class, which is artificially constructed by a set of the poles $\left\{z_{k}\right\}$ as follows:

$$
\begin{aligned}
f_{\text {Carleman }}(z) & =\sum_{k=1}^{K}\left(\frac{1}{1-z_{k} z}+\frac{1}{1-z_{k}^{*} z}\right) e^{-k} \\
& =2 \sum_{n=0}^{\infty} \sum_{k=1}^{K} e^{-k} \cos \left(2 \pi X_{k} n\right) z^{n},
\end{aligned}
$$

where we set the poles at $z_{k}=\exp \left( \pm 2 \pi i X_{k}\right)(k=1,2, \ldots, K)$ on the unit circle. $\left\{X_{k}\right\}$ are i.i.d random variables in the interval $X_{k} \in[0.1]$ and we take $K=100$. Figure 17 shows the absolute values of the residues $\left|A_{k}\right|$ of the Padé approximated functions of order $M=15, M=25$, and $M=45$ for $f_{\text {Carleman }}(z)$. They are arranged in descending order.

As a result, it seems that $\left|A_{k}\right|$ exponentially decreases with a stable exponent, regardless of the order of the Padé approximation. This supports that certainly, the Padé approximation is applicable to the quasianalytic functions in the Gammel conjecture as given in Gammel-Nuttall theorem. The Padé approximation for the quasianalytic function converges to the function even outside the unit circle. It should be also noted that in all cases the tails of $\left|A_{k}\right|$ are rapidly decay because the "truncated" series are essentially analytic functions. 


\section{F. Some Results for Natural Boundary in Noisy Series}

In this appendix some theorems for the random power series are given. See, for example, [21] for the proofs.

Steinhaus's Theorem (1929). Suppose that the power series

$$
f(z)=\sum_{n=0}^{\infty} a_{n} z^{n}
$$

has radius of convergence $R=1$. Let $X_{0}, X_{1}, \ldots, X_{n}$ be a sequence of i.i.d. random variables in the interval $X_{i} \in[0,1]$. Then, with probability one, the random power series

$$
f_{\text {Steinhaus }}(z)=\sum_{n=0}^{\infty} a_{n} w_{n} z^{n}
$$

has a natural boundary on $|z|=1$, where $w_{k}=e^{i 2 \pi X_{k}}$.

Paley-Zygmund Theorem (1932). Suppose that the power series (F.1) has the radius of convergence 1 . Let $r_{0}, r_{1}, \ldots, r_{n}, \ldots$ be a sequence of binary stochastic variables taking -1 or 1 with equal probability. Then, with probability one, the random power series

$$
f_{P-Z}(z)=\sum_{n=0}^{\infty} r_{n} z^{n}
$$

has a natural boundary on the unit circle $|z|=1$.

The similar theorems can hold for random power series $\sum_{n=0}^{\infty} r_{n} z^{n}$ with a sequence of stochastic variables obeying i.i.d. in the interval $r_{i} \in[-1,1]$ or $r_{i} \in[0,1][48]$.

Kahane's Theorem (1985). The circle of convergence is the natural boundary for random Taylor series (F.1) if the coefficients $\left\{a_{n}\right\}$ are independent and symmetric random variables.

The more generalized version has been given in the following form [22].

Breuer-Simon Theorem (2011). Suppose that the power series (F.1) has the convergence radius 1. Then for a.e. $\omega, f(z)=$ $\sum_{n=0}^{\infty} a_{n}(\omega) z^{n}$ has a strong natural boundary on $|z|=1$ if the $a_{n}(\omega)$ is a stationary, ergodic, bounded, and nondeterministic process.

\section{Conflict of Interests}

The authors declare that there is no conflict of interests regarding the publication of this paper.

\section{Acknowledgments}

This paper was partially written for "International Symposium of Complexified Dynamics, Tunnelling and Chaos" held on 2005 in Kusatsu. This work is partly supported by Japanese people's tax via MEXT, and the authors would like to acknowledge them. They are also very grateful to Dr. T. Tsuji and to Koike Memorial House for using the facilities during this study.

\section{References}

[1] G. A. Baker and J. L. Gammel, The Pade Approxima tion in Theoretical Physics, Academic Press, New York, NY, USA, 1970.

[2] G. A. Baker Jr., Essentials of Pade Approximants, Academic Press, New York, NY, USA, 1975.

[3] J. Baker and P. Graves-Morris, Padé Approximants, Cambridge University Press, Cambridge, UK, 2nd edition, 1996.

[4] F. Sasagawa, Scattering Theory, Syouk\&bou, 1991, (Japanese).

[5] H. Stanly, Introduction to Phase Transitions and Critical Phenomena, Clarendon Press, Oxford, UK, 1971.

[6] C. N. Yang and T. D. Lee, "Statistical theory of equations of state and phase transitions. I. Theory of condensation," Physical Review, vol. 87, p. 404, 1952.

[7] B. Nickel, "On the singularity structure of the 2D Ising model susceptibility," Journal of Physics A, vol. 32, no. 21, pp. 38893906, 1999.

[8] R. Kubo, M. Toda, and N. Hashitsume, Statistical Physics II, Springer, Berlin, Germany, 1985.

[9] B. M. McCoy, "Do hard spheres have natural boundaries?", http://arxiv.org/abs/cond-mat/0103556.

[10] D. Bessis, "Padé approximations in noise filtering," Journal of Computational and Applied Mathematics, vol. 66, no. 1-2, pp. 8588, 1996.

[11] H. Stahl, "The convergence of diagonal Padé approximants and the Padé conjecture," Journal of Computational and Applied Mathematics, vol. 86, no. 1, pp. 287-296, 1997.

[12] J. Gilewicz and M. Pindor, "Padé approximants and noise: a case of geometric series," Journal of Computational and Applied Mathematics, vol. 87, no. 2, pp. 199-214, 1997.

[13] D. Bessis and L. Perotti, "Universal analytic properties of noise: introducing the J-matrix formalism," Journal of Physics A, vol. 42, no. 36, 2009.

[14] L. A. Barbosa Coelho and L. A. Baccala, "Pade approximations as a modal identification technique," in Proceedings of the 27th IMAC, Orlando, Fla, USA, February 2009.

[15] A. Berretti and L. Chierchia, "On the complex analytic structure of the golden invariant curve for the standard map," Nonlinearity, vol. 3, no. 1, pp. 39-44, 1990.

[16] C. Falcolini and R. de la Llave, "Numerical calculation of domains of analyticity for perturbation theories in the presence of small divisors," Journal of Statistical Physics, vol. 67, no. 3-4, pp. 645-666, 1992.

[17] R. de la Llave and S. Tompaidis, "Computation of domains of analyticity for some perturbative expansions of mechanics," Physica D: Nonlinear Phenomena, vol. 71, no. 1-2, pp. 55-81, 1994.

[18] A. Berretti and S. Marmi, "Scaling, perturbative renormalization and analyticity for the standard map and some generalizations," Chaos, Solitons and Fractals, vol. 5, no. 2, pp. 257-269, 1995.

[19] A. Berretti, C. Falcolini, and G. Gentile, "Shape of analyticity domains of Lindstedt series: the standard map," Physical Review E, vol. 64, no. 1, Article ID 015202, 2001.

[20] T. W. Korner, Exercises for Fourier Analysis, Cambridge University Press, Cambridge, UK, 1993.

[21] R. Remmert, Classical Topics in Complex Function Theory, Springer, New York, NY, USA, 1st edition, 1998.

[22] J. Breuer and B. Simon, "Natural boundaries and spectral theory," Advances in Mathematics, vol. 226, no. 6, pp. 4902-4920, 2011. 
[23] O. Knill and J. Lesieutre, "Analytic continuation of Dirichlet series with almost periodic coefficients," Complex Analysis and Operator Theory, vol. 6, no. 1, pp. 237-255, 2012.

[24] O. Costin and M. Huang, "Behavior of lacunary series at the natural boundary," Advances in Mathematics, vol. 222, no. 4, pp. 1370-1404, 2009.

[25] W. H. Press, S. A. Teukolsky, W. T. Vetterling, and B. P. Flannery, Numerical Recipes in C, Cambridge University Press, 1988.

[26] W. H. Press and S. A. Teukolsky, "Padé approximants," Computers in Physics, vol. 6, p. 82, 1982.

[27] J. Nuttall, “The convergence of Padé approximants of meromorphic functions," Journal of Mathematical Analysis and Applications, vol. 31, no. 1, pp. 147-153, 1970.

[28] C. Pommerenke, "Padé approximants and convergence in capacity," Journal of Mathematical Analysis and Applications, vol. 41, no. 3, pp. 775-780, 1973.

[29] H. Stahl, "Spurious poles in Padé approximation," Journal of Computational and Applied Mathematics, vol. 99, no. 1-2, pp. 511-527, 1998.

[30] P. Gonnet, R. Pachon, and L. N. Trefethen, "Robust rational interpolation and least-squares," Electronic Transactions on Numerical Analysis, vol. 38, pp. 146-167, 2011.

[31] P. Gonnet, S. Güttel, and L. N. Trefethen, "Robust Padé Approximation via SVD," SIAM Review, vol. 55, no. 1, pp. 101-117, 2013.

[32] H. S. Yamada and K. S. Ikeda, "Analyticity of quantum states in one-dimensional tight-binding model," The European Physical Journal B, 2014.

[33] E. B. Saff and R. S. Varga, "On the zeros and poles of Padé approximants toez," Numerische Mathematik, vol. 25, no. 1, pp. $1-14,1975$.

[34] M. Kac, "On the average number of real roots of a random algebraic equation," Bulletin of the American Mathematical Society, vol. 49, pp. 314-320, 1943.

[35] P. Erdos and P. Turan, "On the distribution of roots of polynomials," Annals of Mathematics, vol. 51, pp. 105-119, 1950.

[36] F. Amoroso and M. Mignotte, "On the distribution of the roots of polynomials," Annales de l'Institut Fourier, vol. 46, no. 5, pp. 1275-1291, 1996.

[37] A. Odlyzko and B. Poonen, "Zeros of polynomials with 0, 1 coefficients," L'Enseignement Mathématique, vol. 39, pp. 317348, 1993.

[38] B. Simon, Orthogonal Polynomials on the Unit Circle Part $l$ :Classical Theory, American Mathematical Society, 2004.

[39] B. Simon, Orthogonal Polynomials on the Unit Circle Part 2 :Spectral Theory, American Mathematical Society, Providence, RI, USA, 2004.

[40] Y. Peres and B. Virág, "Zeros of the i.i.d. Gaussian power series: a conformally invariant determinantal process," Acta Mathematica, vol. 194, no. 1, pp. 1-35, 2005.

[41] B. Simon, Szego's Theorem and Its Descendants: Spectral Theory for L2 Perturbations of Orthogonal Polynomials, Princeton University Press, 2010.

[42] J. Gilewicz and Y. Kryakin, "Froissart doublets in Padé approximation in the case of polynomial noise," Journal of Computational and Applied Mathematics, vol. 153, no. 1-2, pp. 235-242, 2003.

[43] X. Ding and Y. Xiao, "Natural boundary of random Dirichlet series," Ukrainian Mathematical Journal, vol. 58, no. 7, pp. 11291138, 2006.
[44] "We can also define the $Z$-transform by negative power $z^{-n}$. Then the function $Z(z)$ is analytic in outer domain of $|z|=1$, the poles corresponding to damping oscilla tions appear in the inside the unit circle $|z|<1$ ".

[45] J. L. Gammel and J. Nuttall, "Convergence of Padé approximants to quasianalytic functions beyond natural boundaries," Journal of Mathematical Analysis and Applications, vol. 43, no. 3, pp. 694-696, 1973.

[46] A. Shenitzer and N. Luzin, "Function: part I," The American Mathematical Monthly, vol. 105, no. 1, pp. 59-67, 1998.

[47] N. Luzin, "Function: Part II," The American Mathematical Monthly, vol. 105, no. 3, pp. 263-270, 1998.

[48] J.-P. Kahane, Some Random Series of Functions, vol. 5 of Cambridge Studies in Advanced Mathematics, Cambridge University Press, Cambridge, UK, 2nd edition, 1985. 


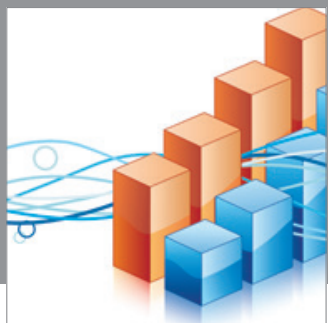

Advances in

Operations Research

mansans

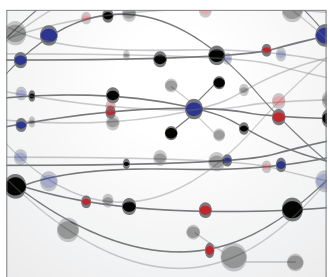

The Scientific World Journal
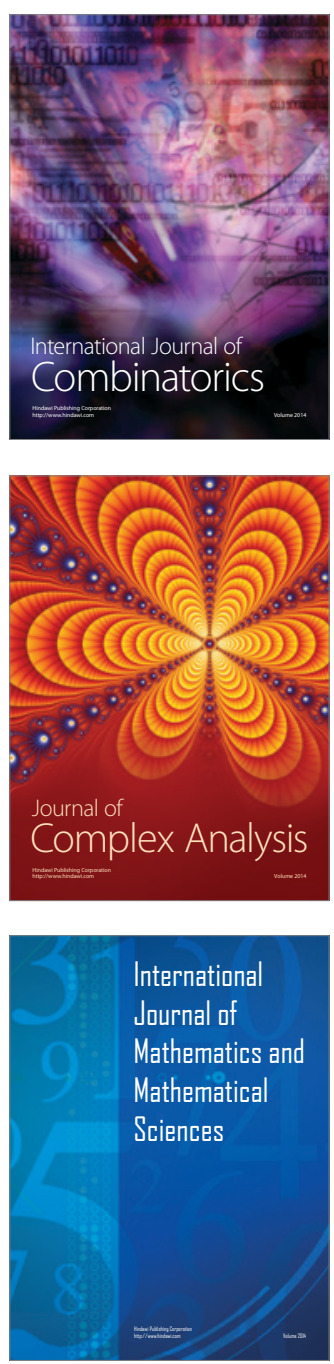
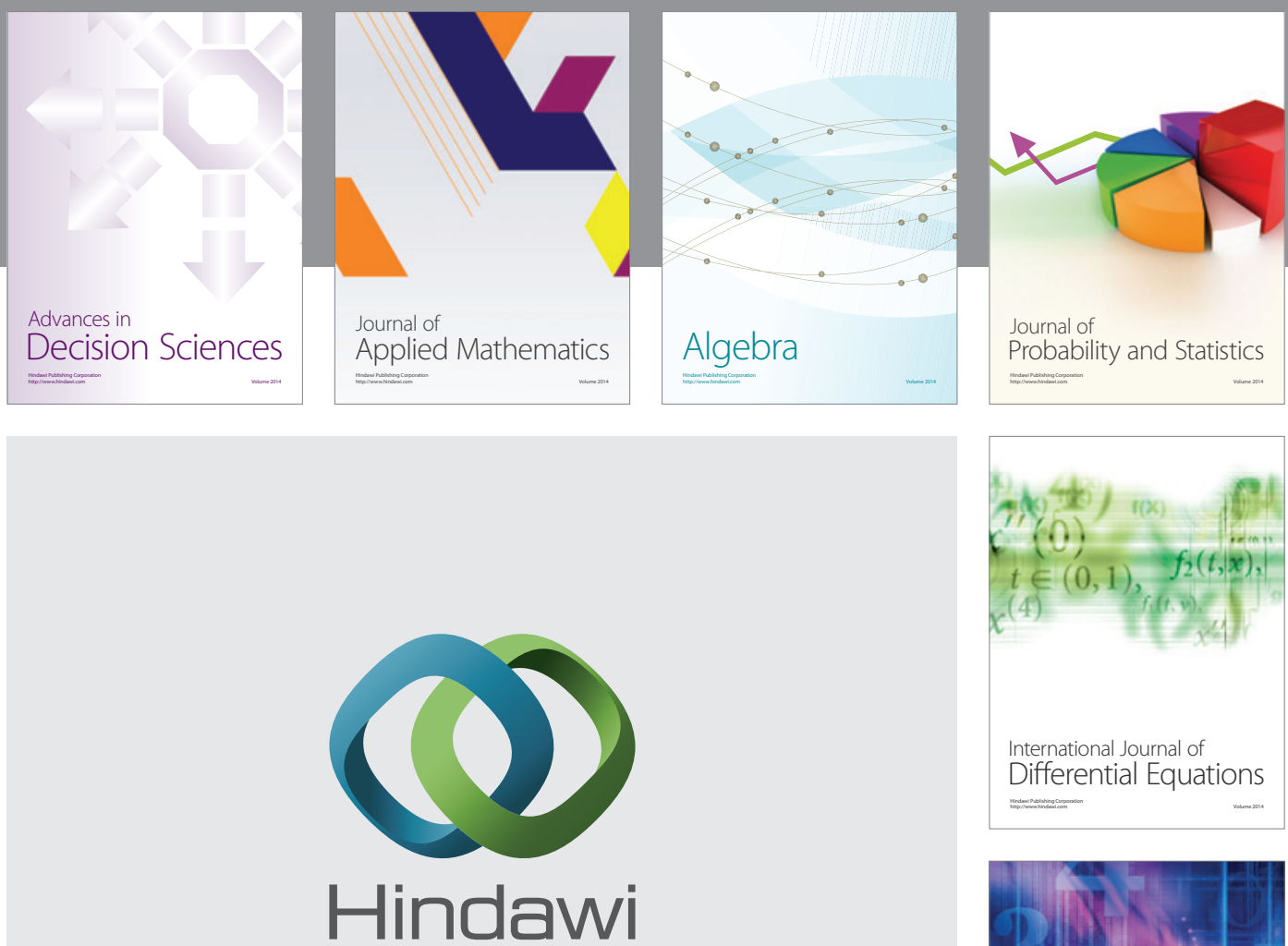

Submit your manuscripts at http://www.hindawi.com
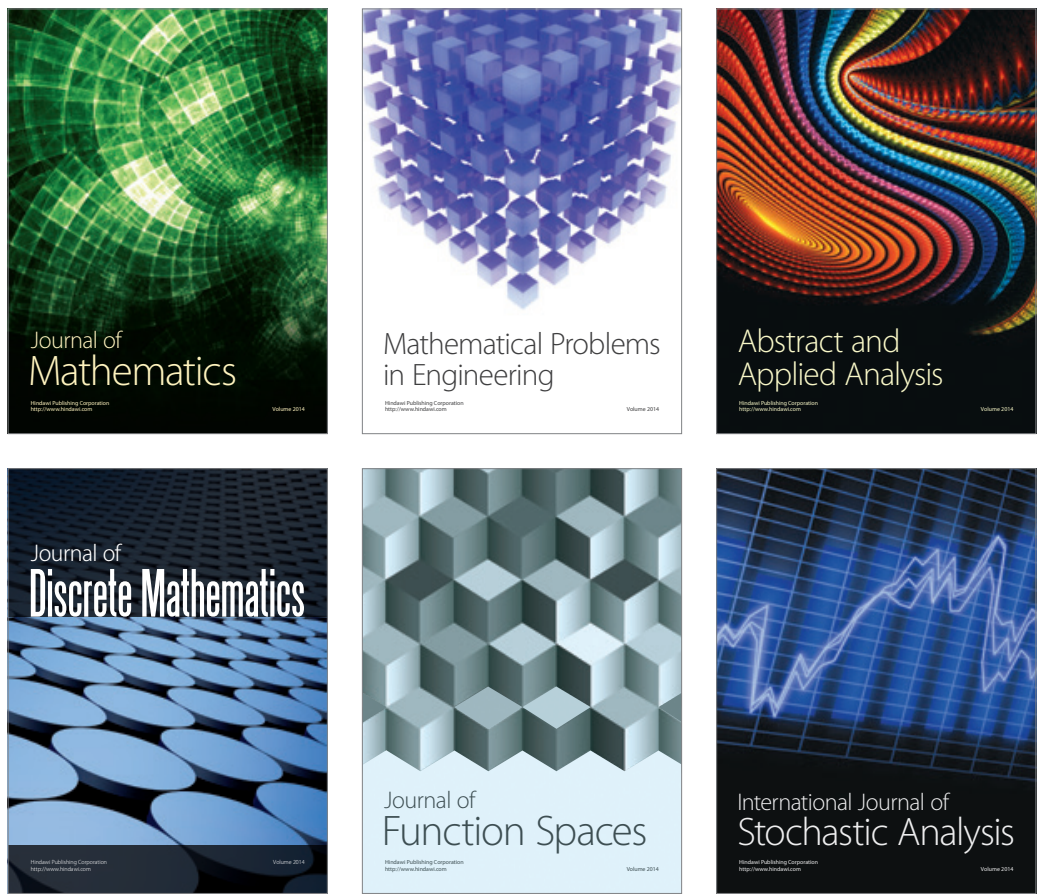

Journal of

Function Spaces

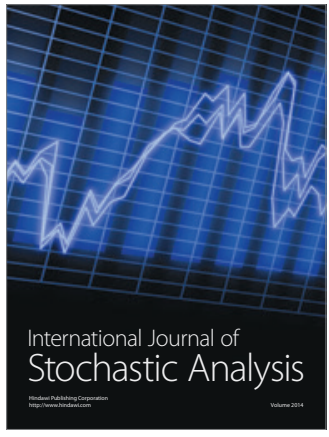

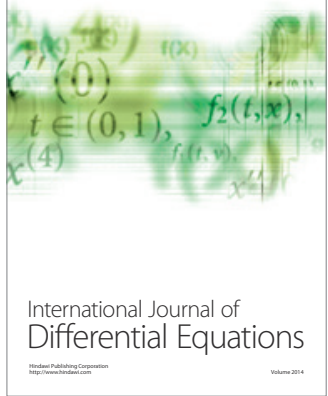
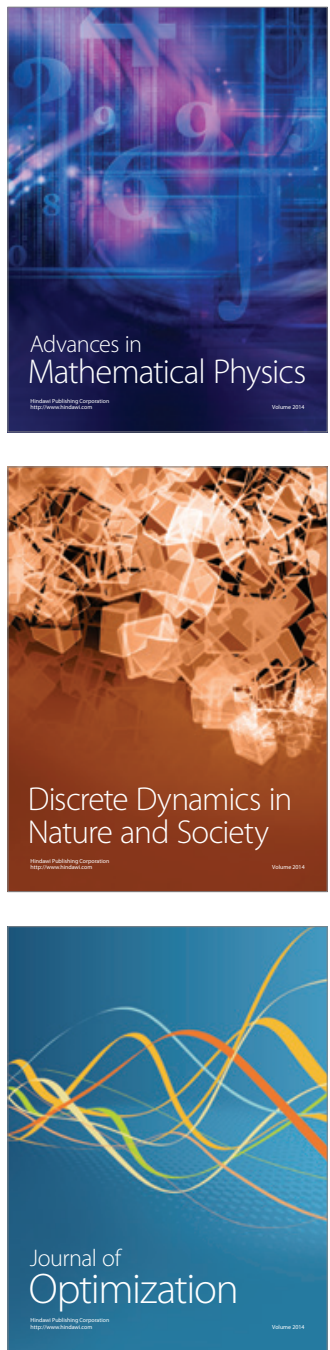